Annu. Rev. Pathol. Mech. Dis. 2020. 15:X-X

https://doi.org/10.1146/annurev-pathmechdis-012419-032742

Copyright $(\mathcal{C} 2020$ by Annual Reviews.

All rights reserved

Mondino et al.

www.annualreviews.org • Legionella and Legionnaires' disease

\title{
Legionnaires' Disease: State of the Art Knowledge of Pathogenesis Mechanisms of Legionella
}

\author{
Sonia Mondino, ${ }^{1}$ Silke Schmidt,,${ }^{1,2}$ Monica Rolando, ${ }^{1}$ Pedro Escoll, ${ }^{1}$ Laura Gomez- \\ Valero, ${ }^{1}$ and Carmen Buchrieser ${ }^{1}$ \\ 'Institut Pasteur, Biologie des Bactéries Intracellulaires, CNRS UMR 3525, 75015 Paris, France; \\ email: smondino@pasteur.fr, silke.schmidt@ pasteur.fr, mrolando@pasteur.fr, pedro.escoll- \\ guerrero@pasteur.fr, Igomez@pasteur.fr, cbuch@pasteur.fr
}

${ }^{2}$ Sorbonne Université, Collège doctoral, 75005 Paris, France

\section{Keywords}

Legionella, Legionnaires' disease, free-living amoeba, eukaryotic-like proteins

\begin{abstract}
Legionella species are environmental gram-negative bacteria able to cause a severe form of pneumonia in humans known as Legionnaires' disease. Since the identification of Legionella pneumophila in 1977, four decades of research on Legionella biology and Legionnaires' disease have brought important insights into the biology of the bacteria and the molecular mechanisms that these intracellular pathogens use to cause disease in humans. Nowadays, Legionella species constitute a remarkable model of bacterial adaptation, with a genus genome shaped by their close coevolution with amoebae and an ability to exploit many hosts and signaling pathways through the secretion of a myriad of effector proteins, many of which have a eukaryotic origin. This review aims to discuss current knowledge of Legionella infection mechanisms and future research directions to be taken that might answer the many remaining open questions. This research will without a doubt be a terrific, scientific journey worth taking.
\end{abstract}




\section{INTRODUCTION}

\subsection{The History of Legionella and Legionnaires' Disease}

Legionella species are gram-negative bacteria that were unrecognized until the summer of 1976 when an explosive outbreak of pneumonia in Philadelphia, Pennsylvania, United States, caught the attention of the US Centers for Disease Control and Prevention (CDC) and the media. An unusual respiratory disease affected 221 attendees of the 58th annual convention of the American Legion, and 34 fatal cases were reported (1). Due to the importance of the outbreak and the fact that the causative agent was not known, the CDC employed what at that time was the largest team in its history to identify the source of the infection. In December 1976, Joseph E. McDade and Charles C. Shepard identified a bacterium as the causative agent of Legionnaires' disease. They discovered a new rod-shaped gram-negative bacterium, named Legionella pneumophila after the American Legion, and the new genus named Legionella, which at that time had only one known species $(\underline{1}-\underline{3})$.

Once the organism was identified, further studies revealed that Legionella had been already isolated in 1947, but it was not further characterized at that time (4). It was also shown that Legionella were the cause of previously unexplained outbreaks of flu-like disease such as the one that occurred in 1968 in Pontiac, Michigan, a clinical condition subsequently named Pontiac fever (ㅁ). Today, the genus Legionella comprises more than 65 different species, and our understanding of the biology and pathogenicity of the different members of this genus continues to increase.

\subsubsection{Ecology and epidemiology}

Legionella are gram-negative rod-shaped $\gamma$-proteobacteria that are ubiquitously found in freshwater environments, as well as in moist soil and composted material (ㅁ). Legionella was the first bacterium described that multiplied within protozoan hosts, primarily aquatic amoebae, which led to the idea that the capacity of the bacteria to infect protozoa may also allow them to replicate within human lung macrophages ( 7 ), a finding that was confirmed later through many different studies (reviewed in Reference $\underline{8}$ ). Today, it is established that Legionella are primarily found in the environment, either associated with their host or as free-living biofilm-associated bacteria ( ) (Figure 1).

<COMP: PLEASE INSERT FIGURE 1 HERE> 
Figure 1 Transmission routes and life cycle of Legionella pneumophila and L. longbeachae. Legionella are commonly found in freshwater environments associated with biofilms or replicating inside amoebae. The development of manmade aquatic environments, such as showers, cooling towers, and fountains, allows for bacterial distribution and amplification in these artificial environments. Subsequent aerosolization from these sources exposes humans to inhalation or aspiration of contaminated water droplets. Through this means, Legionella can reach the human lungs, where they can infect alveolar macrophages using the same mechanisms that they utilize to survive within their amoebal hosts. Inside the host cell, Legionella reside in a separated compartment, from where they modulate diverse host signaling pathways through the secretion of effector proteins by a dedicated Dot/Icm type 4B secretion system. L. longbeachae is found in soil and potting mixes, some of which contain composted pine bark, and, presumably, is also associated with amoebae and biofilm communities. The route of human infection with $L$. longbeachae has not yet been established, but it may involve the inhalation of aerosolized particles generated after the manipulation of contaminated soil-derived products. Abbreviations: ER, endoplasmic reticulum; G, Golgi apparatus; LCV, Legionella-containing vacuole; N, nucleus.

Human infection most commonly occurs as a consequence of inhaling Legionella-containing aerosols generated by contaminated manmade water sources, such as showers, hot tubs, plumbing networks, and air-conditioning systems. However, aspiration of contaminated water has been suggested as another route of transmission ( $\underline{10})$ (Figure 1). Although human-to-human transmission was not thought to occur, one case has been reported, suggesting that this form of transmission may exist, but it is rare (11). In general, human infection is incidental and a dead end for the bacteria. Individuals at higher risk for developing Legionnaires' disease are males older than 50 years, smokers, and people with an underlying medical condition such as diabetes, cancer, or immunosuppression; however, anybody can develop Legionnaires' disease (12). Summer and early fall are the most common times of the year for Legionella infection to occur.

The burden of Legionnaires' disease in Europe and in the United States is increasing each year, with both regions showing comparable notification rates and similar settings and epidemiology of infections. The increase in reported cases could be due to environmental conditions, such as changes in rainfall, temperature, and climate, that can affect the incidence $(\underline{13})$; to the increasing proportion of more susceptible people, such as elderly people and those who are immunocompromised; and partly also to improvements in the surveillance systems in these regions over the past two decades $(\underline{14})$.

To put Legionnaires' disease in perspective, from 2011 to 2015, the age-standardized rate of Legionnaires' disease in Europe showed an average annual increase of 0.09 cases per 100,000 
individuals, reaching 1.30 cases per 100,000 individuals in 2015 . The mortality rate fluctuated between 0.07 and 0.09 deaths per 100,000 individuals, with an overall case-fatality ratio continuously decreasing during the 2011-2015 period. The decreasing case-fatality ratio may be due to improvements in reporting completeness that may be correcting a former bias toward fatal outcomes of the disease (14). During this time, the source of infection was identified for $88 \%$ of reported cases. Of these, $70.7 \%$ of infections were community acquired; $19.9 \%$ were travel associated; and 7.3\% were health-care related (14). In addition, a report by the European Centre for Disease Prevention and Control in 2016 showed 1.4 case notifications per 100,000 individuals, the highest ever observed for Europe, with a case-fatality ratio similar to the one observed in 2015 (15). In accordance with reports from Europe, active surveillance in the United States described an incidence of 1.89 cases of Legionnaires' disease per 100,000 individuals in 2015, with a case-fatality ratio similar to that observed in Europe and with similar epidemiology and sources of infection (16).

To date, the genus Legionella comprises 65 species, but, interestingly, not all of them are equally responsible for the laboratory-confirmed cases of Legionnaires' disease worldwide, as $L$. pneumophila accounts for $80-90 \%$ of the cases in Europe and the United States (17). Furthermore, even within this species, disease-causing L. pneumophila strains are unevenly distributed, as strains of serogroup ( $\mathrm{Sg}$ ) 1 are responsible for approximately $90 \%$ of cases. Additionally, within the $\mathrm{Sg} 1$ strains, specific clones have recently emerged and already account

for more than 50\% of the reported cases of Legionnaires' disease in northern Europe, suggesting that these disease-related clones became adapted to manmade aquatic environments (18). $L$. longbeachae accounts for approximately $1 \%$ of cases worldwide, but, interestingly, for $50-60 \%$ of cases in Australia and New Zealand. However, during the past 10 years, cases caused by $L$. longbeachae infection have also been increasingly reported in Europe (19). Other species and serogroups, such as L. pneumophila $\mathrm{Sg} 3$ and $\operatorname{Sg} 6$, L. bozemanii, and L. micdadei, may also cause disease in Europe and the United States, but are rare $(\underline{14}, \underline{17}, \underline{20})$.

\subsubsection{Detection and treatment}

For Legionella infection, the time to detection remains critical for the final disease outcome, especially for at-risk populations. A patient with community-acquired Legionnaires' disease generally shows pneumonic as well as extrapulmonary findings, such as gastrointestinal and neurological symptoms, relative bradycardia, hypophosphatemia, or increased serum ferritin 
levels $(\underline{21})$, or some combination of these. In addition to the clinical symptoms, laboratory confirmation is essential for diagnosis; thus, specific detection methods have been developed for assessing Legionella infection using sputum or respiratory secretions; tissue, blood, or serum samples; or urine samples (22). These methods include serological and antibody-based assays, bacterial culture, urinary antigen tests, and nucleic acid amplification testing (for detailed reviews see References $\underline{22}, \underline{23}$ ).

Initially, serology was the method of choice to assess infections with Legionella, but the use of this technique has dropped significantly because of the development of more user-friendly and rapid methods, such as the urinary antigen test and polymerase chain reaction (PCR)-based detection methods. However, serology remains relevant for retrospective epidemiological investigations and when the infectious agent cannot be isolated despite strong evidence of Legionnaires' disease (22). The urinary antigen test, which detects a component of the Legionella cell wall in urine samples, is now widely used as a first-line screening method because it is easy and low cost, and results are rapidly available. However, as it allows only for the detection of L. pneumophila $\mathrm{Sg} 1$, there is still a need to develop assays that identify different serogroups and Legionella species $(\underline{22}, \underline{24})$. Recently, PCR-based methods, such as the ones developed to detect L. pneumophila Sg1 (25) or the emerging L. pneumophila ST47 clone (26), have become more commonly used in reference centers, but with the exception of New Zealand, they are still used only rarely for clinical diagnosis (27). The utility of PCR-based assays to complement other diagnostic methods has also been demonstrated by the development of a rapid and reliable multiplexed real-time PCR assay that allows for the detection of four clinically relevant non-pneumophila species from mock human sputum specimens $(\underline{28}, \underline{29})$. Nevertheless, culture on defined growth medium remains the standard reference method for Legionella diagnosis and identification, as it allows for identification of different Legionella species and serogroups, and subsequent epidemiological studies of their distribution (23) .

Fortunately, antibiotic resistance is not yet a problem for L. pneumophila infections. To date, one fluoroquinolone (ciprofloxacin)-resistant L. pneumophila strain has been isolated from a patient with Legionnaires' disease in the Netherlands ( $\underline{30})$, and the in vivo selection of fluoroquinolone resistance mutations in L. pneumophila was reported in two infected patients treated with these antibiotics in France (31), suggesting that, overall, antibiotic resistance is rare. Nevertheless, the incidence of fluoroquinolone resistance might be underestimated, supporting 
the need for prompt identification of Legionella infection to ensure the rapid and accurate administration of antibiotic therapy ( $\underline{32})$. Related to this, a digital PCR assay used to detect fluoroquinolone-resistant mutants of Legionella in patients' samples has proven useful as a diagnostic tool to assess the effectiveness of antibiotic therapy (32). Given the rare instances of resistance reported, the recommended antimicrobial therapy still includes fluoroquinolones (ciprofloxacin, levofloxacin, or moxifloxacin) or macrolides (azithromycin) (33).

\subsection{Legionella longbeachae: Similar but Different}

L. longbeachae is a major cause of disease only in Australia and New Zealand (20). However, over the past decade, infections with this bacterium have also been increasingly reported from

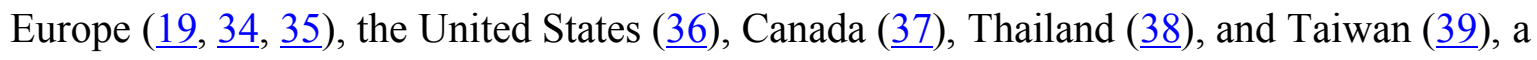
phenomenon that might correlate with increased clinical awareness and the wider use of improved detection methods. A total of 15 serogroups are recognized for L. pneumophila, but only 2 are recognized for L. longbeachae, with $\mathrm{Sg} 1$ being responsible for the majority of reported cases. A comparison of the clinical features and outcomes of disease caused by $L$. pneumophila and L. longbeachae showed that both species cause a similar disease pattern, and similar risk factors apply, such as older age, being a smoker, and having immunosuppression or other preexisting medical conditions. However, the main seasons for disease caused by $L$. longbeachae are spring and summer, whereas L. pneumophila legionellosis occurs more

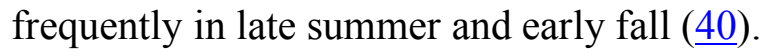

Legionella species are ubiquitously found in aquatic environments; however, L. longbeachae is found in moist soil and potting mixes, presumably also associated with protozoa. Thus, gardening and using potting soil are unique risk factors associated with L. longbeachae infections (41). This characteristic might partly explain the differences in the seasons of onset, as gardening activities usually occur more frequently in spring and summer. The route of transmission to humans is still not completely understood, but it may be that infection occurs through the inhalation of aerosolized, contaminated compost particles that are formed when the bags are opened, when the potting mix is handled, or when plants are watered $(\underline{20}, \underline{41})$ (Figure 1). Yet the report of a recent outbreak suggested that waterborne transmission of L. longbeachae may also occur, as the bacterium was detected both in the water of a cooling tower and as cause of human infection. However, due to the lack of a clinical isolates, the cooling tower could not be confirmed as the source of this infection (느). 
L. pneumophila has a pronounced, so-called biphasic life cycle during which it switches between a replicative (avirulent) and a transmissive (virulent) form (43). This differentiation, in which metabolic as well as morphogenetic changes take place, occurs during the transition between intracellular and extracellular environments, and it is accompanied by a specific switch in the gene expression pattern (44). In a simple model, when conditions are favorable for replication (in a nutrient-rich environment), L. pneumophila represses the expression of the transmission traits (motility, osmotic- and acid-resistance, cytotoxicity) and expresses the genes necessary to replicate and multiply intracellularly and to use the resources available from the host. Conversely, when the bacteria density increases and nutrients become limited, $L$. pneumophila stops replicating, while inducing the coordinated expression of the transmission traits (45). Thus, the bacteria escape from the cell and spread to new hosts to resume the cycle. During bacterial growth in liquid medium, the replicative and transmissive phases are represented by, respectively, the exponential and stationary growth phases $(\underline{43}, \underline{46})$. As a consequence of this biphasic life cycle, the infection of a host cell and survival of $L$. pneumophila inside the cell depend on its metabolic state (47). A key regulator of the switch between these two phases is carbon storage regulator A (CsrA), an RNA-binding protein that is a global repressor of the transmission genes during the replicative phase $(\underline{47}, \underline{48})$. Its repressive function is relieved under starvation conditions, as limited amino acid availability signals the production of the alarmone guanosine pentaphosphate [(p)ppGpp], which leads to the activation of the two-component system Legionella transmission activator and sensor (LetA/LetS) and the alternative sigma factor RNA polymerase sigma factor (RpoS). These regulators activate transcription of the small noncoding RNAs RsmX, - Y, and $-Z$ that sequester CsrA, thereby releasing the repression of the transmissive traits $(\underline{49}, \underline{50})$. A genome-wide analysis of CsrA targets provided evidence that this protein impacts the central carbon metabolism, motility, and infective capacity of $L$. pneumophila by controlling the expression of at least $40 \mathrm{Dot} / \mathrm{Icm}$ type 4B secretion system (T4SS) effector proteins (51). Comparable to L. pneumophila, L. longbeachae encodes the LetA/LetS two-component system and a CsrA protein that shows $98 \%$ amino acid similarity with the L. pneumophila CsrA; however, transcriptome analyses have shown that this species does not undergo as dramatic a switch between the two phases as does L. pneumophila $(\underline{50}, 52)$. These findings are in line with the observation that the infective capacity of $L$. longbeachae seems to be independent of its growth phase ( $\underline{53})$. 
Further differences between L. pneumophila and L. longbeachae were identified when the genome sequence of $L$. longbeachae was analyzed $(\underline{52}, \underline{54})$. Particularly interesting was the presence of a largely different T4SS effector repertoire, as only about $30 \%$ of the effectors present in L. pneumophila were also present in L. longbeachae $(\underline{52}, \underline{55})$. Also, while $L$. pneumophila is non-encapsulated and flagellated, L. longbeachae encodes for a capsule but not for flagella ( $\underline{52}$ ). Actually, the presence of cytosolic flagellin leads to clearance of $L$. pneumophila from mouse macrophages due to the activation of the Naip5-Nlrc4 inflammasome and subsequent cell death by pyroptosis ( $(\underline{6})$. Mice are more susceptible to L. longbeachae infection, even when compared to a $L$. pneumophila mutant lacking flagella, suggesting that the high lethality and the poor stimulatory activity of $L$. longbeachae could also be a consequence of the presence of a capsule as well as the different reservoir of effectors $(\underline{52}, 57)$. Overall, clear phenotypic differences are evident between these two species, and yet little is known about $L$. longbeachae's biology and infection processes.

\section{LEGIONELLA: AN ARMY WITH A LARGE ARSENAL OF WEAPONS}

Legionella are able to replicate in a wide variety of phagocytic hosts, ranging from numerous amoeba species to mammalian cells ( $\underline{8})$, in which they form a distinct membrane-bound replicative niche known as the Legionella-containing vacuole (LCV) (Figure 1). This sophisticated intracellular compartment allows the bacteria to evade phagolysosomal degradation as well as to shelter from intracellular defenses and to intercept nutrients to support replication. In order to do these things, Legionella employ different secretion systems that deliver virulenceassociated proteins across one or two cell membranes to the site of action. While the type 2 secretion system (T2SS) and T4SS are encoded by all Legionella strains, the type 1 secretion system (T1SS) is restricted to L. pneumophila, and the type 4A secretion system (Lvh type) is randomly distributed among different species $(\underline{58}, \underline{59})$. The T2SS and T4SS have been extensively studied in L. pneumophila as they play essential roles during infection.

The delivery of effector proteins via T2SS is a two-step process in which proteins are first transported into the periplasm, where they are recognized by the T2SS apparatus, and then exit through a dedicated pore (무). Subsequently, T2SS effectors may be found associated with the LCV membrane, after they had escaped into the host cytosol (61). This system translocates more than 25 effector proteins ( $\underline{62})$ that play major roles in intracellular replication in amoeba and also 
in L. pneumophila pathogenesis in humans ( $\underline{63})$. One example is a chitinase that is secreted by T2SS that promotes bacterial persistence in the lungs (ㅁ4).

The Dot/Icm T4SS is critical for LCV biogenesis and intracellular replication $(\underline{65}, \underline{66})$.

Recently, it was shown that the L. pneumophila T4SS is located at the bacterial cell poles, and effector delivery is triggered by phagocytosis $(\underline{67}, \underline{68})$. Importantly, T4SS governs all steps of the intracellular life of L. pneumophila by secreting more than 330 effector proteins that target fundamental cellular processes conserved between protozoa and mammals (Table 1) (Figure 2).

\section{<COMP: PLEASE INSERT TABLE 1 HERE $>$ <COMP: PLEASE INSERT FIGURE 2 HERE>}

Figure 2 Intracellular pathways regulated by secreted Legionella pneumophila effectors: representation of the intracellular cycle of $L$. pneumophila and the effectors secreted by the type 4 secretion system that control the cellular response at each step of the cycle. After bacterial uptake (1), L. pneumophila avoids endocytic maturation (2) and instead recruits ER-derived vesicles to the LCV (3), allowing for the formation of a safe niche where (4)-(6) the bacterium replicates and modulates cell signaling pathways. Once the replication cycle is completed, $L$. pneumophila exits the cell (7) and infects a new host. Abbreviations: ER, endoplasmic reticulum; LCV, Legionella-containing vacuole; LY, lysosome.

\subsection{Legionella Successfully Escape Host Cell Degradation}

After bacterial uptake, L. pneumophila avoids endocytic maturation and phagolysosomal degradation. Instead, the bacterium modulates specific host cell signaling pathways by the secretion of a myriad of T4SS-effector proteins, allowing the formation of a safe niche where Legionella can efficiently replicate. During the last two decades, several of these effector proteins have been characterized functionally leading to a better understanding of the mechanisms employed by L. pneumophila to subvert host cell functions. Among those also several novel posttranslational modifications of host proteins induced by a bacterial pathogen have been reported in Legionella for the first time.

\subsubsection{Legionella pneumophila uptake and evasion of the endocytic maturation pathway}

Although the Dot/Icm T4SS seems to promote bacterial uptake into phagocytic cells $(\underline{69}, \underline{70})$, the entry mechanism itself depends on the host cell machinery. L. pneumophila is engulfed by host cells through a phagocytic and macropinocytic phosphatidylinositol $(3,4,5)$ trisphosphate $\left[\operatorname{PtdIns}(3,4,5) \mathrm{P}_{3}\right]$-rich cup (1) $)$ Furthermore, it has been shown that a functional T1SS is also 
required for entry into the host cell (2). Shortly after internalization, phagosomes containing $L$. pneumophila evade endocytic maturation and prevent fusion with lysosomes (푸). $L$. pneumophila prevents vacuolar acidification by blocking the host vacuolar ATPase (v-ATPase), a proton pump present throughout the membranes of the endocytic pathway. This process is driven by two secreted effectors: SidK and WipB. SidK binds the v-ATPase regulatory subunit VatA, resulting in the inhibition of ATP hydrolysis and proton translocation (74). WipB is a lysosome-targeted phosphatase that localizes to acidified LAMP1-positive lysosomal compartments where it interacts with the v-ATPase. SidK and WipB may converge to repress the activity of the host v-ATPase (도).

Phagosome maturation is tightly regulated by T4SS, as the Dot/Icm system appears to redirect vacuoles containing L. pneumophila away from the canonical endocytic pathway at an extremely early stage of infection. Small GTPases of the Rab family represent an important group of proteins involved in phagosome maturation, and the binding of specific Rab proteins to intracellular organelles enables specific targeting. Proteomic analyses of purified LCVs revealed the presence of several small GTPases anchored to the pathogen vacuole: Rab5, Rab7, Rab14, and Rab21 ( 16 , 77). The GTPase Rab5 is an important regulator of the early endocytic pathway: GTP-bound Rab5 orchestrates the recruitment of several downstream ligands, resulting in PtdIns(3)P-mediated recruitment of early endosomal antigen 1 (EEA1) (므). Interestingly, a secreted effector named VipD has been shown to exhibit phospholipase A1 activity that is activated only upon binding to endosomal Rab5. VipD thus localizes to endosomes and can catalyze the removal of PtdIns(3)P from endosomal membranes. Consequently, EEA1 and other transport and fusion factors are depleted from endosomes, rendering them fusion incompetent (79). PieE, another secreted effector, has been shown to bind both Rab5 and Rab7, but its

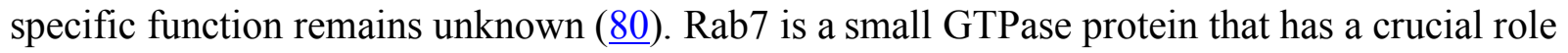
during phagosome maturation, as it gradually replaces Rab5 to induce the fusion between the degradative late endosomes and lysosomes (11). Rab5 and Rab7 also play roles in regulating retrograde trafficking, connecting the endosomal system with the trans-Golgi network ( $\underline{82}) . L$. pneumophila affects this trafficking pathway through the secreted effector RidL ( $\underline{83})$.

\subsubsection{Legionella pneumophila interaction with the endoplasmic reticulum and formation of Legionella-containing vacuoles}

Intercepting vesicular traffic from endoplasmic reticulum (ER) exit sites and vesicle budding 
from the ER appear to be required for the establishment of the replication vacuole ( $\underline{84})$. In particular, it has been proposed that the LCV is localized in proximity to the ER exit sites, ideally suited to hijack vesicle trafficking from the retrograde secretory pathway on the route to the Golgi compartment (ㅁ5). The small GTPases Arf1, Sar1, and Rab1 are important molecules that regulate host vesicular and membrane transport processes; during L. pneumophila infection they participate in the recruitment of ER-derived vesicles to the LCV membrane. Rab1 recruitment to the LCV is a well-orchestrated T4SS-dependent process that has been extensively studied. Indeed, Rab1 is a direct target of several different secreted effectors. (a) SidM (DrrA) is a protein containing three functional domains: a C-terminal domain that binds PtdIns(4)P ( $\underline{86})$, thereby also representing an LCV marker that accumulates on the membrane of the pathogen compartment; a guanine nucleotide exchange factor and a guanine nucleotide dissociation inhibitor displacement factor that recruit $\operatorname{Rab} 1$ to the $\operatorname{LCV}(\underline{87}, \underline{88})$; and an N-terminal enzymatic domain that catalyzes the addition of adenosine monophosphate to Rab1 (AMPylation) (모, 90). (b) SidD has been characterized as a deAMPylase that removes the adenosine monophosphate moiety from Rab1 (91). (c) LepB encodes for a GTPase-activating protein that converts Rab1 GTP into Rab1 GDP (2) . (d) The phosphocholinase AnkX attaches a phosphocholine moiety to Rab1, thus disrupting secretory trafficking (푸). (e) Lem3 removes this phosphocholination (94). And, finally, $(f)$ LidA has Rab1-binding activity and facilitates the tethering of ER-derived vesicles $(\underline{90})$. Thus, the recruitment and functional modifications of Rab1 facilitate the recruitment of ER-derived vesicles to the phagosome membrane.

The function of the small GTPases Arf1 and Sar1 is also important for the recruitment and tethering of ER vesicles to the LCV (푸). Arf1 has been shown to play a critical role in coat protein complex (COP) I-mediated retrograde trafficking in eukaryotic cells, whereas Sar1 is involved in intracellular COPII-mediated protein trafficking from the ER to the Golgi apparatus. The secreted effector RalF is a guanine nucleotide exchange factor that directly activates and recruits Arf1 to the LCV membrane (도). Interestingly, it has been suggested that because the bacteria enter at the cellular periphery, where the ER interacts with the plasma membrane (므), the first microbial encounter would be with tubular peripheral ER. This was confirmed by the observation that the secreted effector Ceg9 directly associates with Rtn4, a protein that regulates ER tubule formation ( $\underline{97})$.

The LCV fuses with the ER by a noncanonical pairing of the vesicular membrane SNARE 
protein Sec22b on ER-derived vesicles with a plasma membrane target SNARE complex containing host syntaxins (요). SNAREs are host proteins that directly facilitate membrane fusion events (99). The SidM (DrrA) effector is sufficient to stimulate SNARE-dependent membrane fusion within Rab1 activation (100). Nonetheless, L. pneumophila also encodes for a secreted effector, LseA, that acts as a SNARE protein, suggested to mediate membrane fusion events in Golgi-associated pathways (101). Additionally, the $\mathrm{LegC} 3$ effector has also been referred to as a SNARE-like protein, which can form a SNARE-like hybrid complex with VAMP4 and modulate membrane fusion events (102) (Please add REF 102a: Shi X, Halder P, Yavuz H, Jahn R, Shuman HA. 2016. Direct targeting of membrane fusion by SNARE mimicry: Convergent evolution of Legionella effectors. PNAS. 113(31):8807-12).

The LCV is also able to move along microtubules, thanks to the activity of the secreted effector LegG1 (MitF), which activates Ran GTPase, thus promoting LCV formation, microtubule stabilization, and LCV motility (103). Interestingly, it has been recently shown that the T4SS effector LegG1 (MitF) is also implicated in mitochondrial fragmentation during infection that depends on the host factors DNM1L, Ran, and RanBP2 by a mechanism that, although not yet elucidated, has been suggested to involve WASP-Arp2/3-mediated recruitment of DNM1L to mitochondria. Legionella-induced mitochondrial fragmentation leads to a Warburg-like metabolism in the host cell that promotes pathogen replication $(\underline{104}, \underline{105})$.

\subsubsection{Legionella pneumophila modulation of host cell signaling pathways}

The transformation of the nascent phagosome into a vacuole derived from the ER resembles an immature autophagosome. Indeed, it has been shown that the LCV carries markers associated with autophagosomes (106) and that several T4SS effectors play roles in inhibiting the autophagic response of the host cell to avoid the degradation of the vacuole by the autophagy machinery: (a) RavZ interferes with autophagy by irreversibly deconjugating an autophagyrelated ubiquitin-like protein, LC3, from phosphatidylethanolamine (107); (b) Lpg1137 targets the mitochondria-associated ER membranes (MAMs) and cleaves syntaxin 17, a SNARE implicated in autophagy, via its Ser protease activity, thereby blocking the process $(\underline{108)}) ;(c)$ $L p$ SPL, another MAM-located effector, prevents autophagosome formation by disturbing the host's sphingolipid metabolism ( $\underline{109})$.

During its intracellular replication cycle, L. pneumophila continuously interferes with different host cell signaling pathways to hijack the cellular response. An important role in signal 
transduction in mammalian cells is played by the tyrosine kinase machinery, and Src homology 2 domains, sequence-specific phosphotyrosine-binding modules, which are key actors required for substrate recruitment and catalytic activity. Interestingly, L. pneumophila encodes for Src homology 2 domain proteins that can translocate into host cells and bind phosphotyrosine (110). Furthermore, LegK7, a newly described effector kinase, promotes intracellular bacterial growth by targeting the host cell Hippo pathway (111). LegK7, like the Hippo kinase MST1, directly phosphorylates MOB1, thus triggering a signaling cascade that alters the transcriptional landscape of host cells.

Another preferential target of bacterial pathogens is the nuclear factor kappa-light-chain enhancer of activated B cells (NF-kB) pathway, due to its central role in transcriptional regulation and activation of host innate immune responses. It has been observed that $L$. pneumophila infection impacts the NF- $\mathrm{kB}$ pathway in a differential way, depending on the stage of infection (112). LegK1 and LnaB are secreted L. pneumophila effectors that strongly activate the NF- $\mathrm{KB}$ transcription factor $(\underline{113}, \underline{114})$. The activity of MavC $-\mathrm{a}$ transglutaminase that catalyzes monoubiquitination of the E2 enzyme UBE2N, thus inhibiting the formation of Lys63 polyubiquitinated chains - dampens NF- $\mathrm{kB}$ signaling, probably counteracting the effects of NF$\kappa \mathrm{B}$ activation at the initial phase of infection (115). Conversely, the Lgt family of cytotoxic glucosyltransferases Lgt1, -2 , and -3 , together with SidI, and SidL specifically decrease the

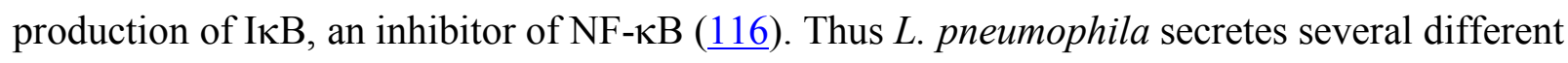
effectors to fine-tune NF- $\mathrm{kB}$ signaling to its advantage.

Similar to the NF-KB pathway, the mitogen-activated protein kinase (MAPK) pathway is also a central signaling cascade that is essential for the activation of immune responses. Indeed, $L$. pneumophila activates this pathway in a T4SS-dependent manner $(117,118)$ by secreting five effectors that inhibit host translation and lead to MAPK activation, thus shaping the transcriptional response of the host cell (119). Another secreted effector, $\mathrm{Ceg} 4$, can modulate the phosphorylation state of eukaryotic MAPKs through its haloacid dehalogenase-like phosphatase domain (120).

L. pneumophila is also able to directly modulate the host's transcriptional machinery by modifying histone marks. The T4SS-secreted L. pneumophila effector RomA methylates Lys14 of histone $\mathrm{H} 3$, a key residue usually acetylated at active promoters, to decrease cellular transcription (121). SnpL, another effector, also targets the host cell nucleus, where it binds the 
eukaryotic transcription elongation factor SUPT5H, which is involved in regulating RNA polymerase II-dependent mRNA processing and elongation (122).

\subsubsection{Legionella pneumophila and interactions with the ubiquitin and apoptotic pathways}

Ubiquitination is an important posttranslational modification in eukaryotic cells that regulates the activity and cellular localization of proteins and affects essential routes, for example, the immune response. Several T4SS effectors of L. pneumophila show similarities to eukaryotic E3 ubiquitin ligases, enzymes that actively participate in protein ubiquitination. LubX and GobX are U-box domain-containing E3 ligases: LubX, structurally similar to the RING E3 ligase domain, directly modifies Cdc2-like kinase 1 (Clk1) (123), whereas the targets of GobX remain to be determined, although its localization to Golgi membranes suggests that it functions at or in close proximity to this compartment (124). RavN encodes an atypical U-box-like motif and possesses E3 ubiquitin ligase activity (125), while SidC, an effector known to enhance ER recruitment to the LCV, (126) defines a unique family of E3 ubiquitin ligases. SidC possesses atypical ubiquitin ligase activity as it uses a Cys-His-Asp triad to catalyze the formation of high-molecular-weight polyubiquitin chains through multiple ubiquitin Lys residues (127). LegU1, LegAU13, and LicA are F-box domain-containing proteins, translocated into the cytosol by T4SS, which specifically interact with components of the host ubiquitination machinery. In addition, LegU1 targets and ubiquitinates the host chaperone BAT3, a protein involved in apoptosis and ER stress response (128). AnkB is another F-box-containing secreted effector that interacts with Skp1 to form a Skp-Cullin-F-box complex that ubiquitinates ParvB (129). AnkB has also been suggested to play a role in supplying the replicative vacuole in amino acids through AnkB-dependent degradation of polyubiquitinated proteins that are used by L. pneumophila as nutrients $(\underline{130})$.

Recently, the members of the SidE effector family (SdeA, SdeB, SdeC, and SidE) were shown to ubiquitinate ER-associated Rab GTPases by a novel ubiquitination mechanism that does not require E1 and E2 enzymes of the host ubiquitination machinery: Ubiquitin is first activated by Arg-ADP ribosylation by the mono-ADP-ribosyltransferase domain of SdeA; the intermediate is then cleaved by the phosphodiesterase domain within the same enzyme; and this occurs concomitant with the attachment of ubiquitin to Ser residues of substrate proteins via a phosphoribosyl linker $(\underline{131}, \underline{132})$. Interestingly, the activity of SidE is affected by SidJ, an effector that reverses the ubiquitination of SidE-modified substrates (133). The members of the SidE family also transfer ubiquitin onto Rtn4 to control tubular ER dynamics (134). LotA 
(Lem21) is another deubiquitinase that was recently discovered and that possesses a Cys protease activity by which it is able to cleave ubiquitin from the LCV (135).

To preserve its replication niche, L. pneumophila modulates host cell-death pathways via the action of several T4SS substrates $(\underline{136})$. SidF directly interacts with and neutralizes proapoptotic BNIP3 and Bcl-rambo (137), whereas SdhA contributes to the prevention of cell death by an unknown mechanism (138). Finally, L. pneumophila also possesses the ability to promote cell death: Several secreted effectors have been shown to induce proapoptotic caspase-3 activity (139). Therefore, fine-tuned control of the secretion of antiapoptotic and proapoptotic effectors might be necessary to support bacterial replication at the beginning of infection and to promote the release of the pathogen from the host cell at the end of the infection cycle.

\subsection{Specific Features of the Legionella Dot/Icm T4SS Effector Repertoire}

It is well established that L. pneumophila delivers more than 330 effector proteins into its host cells $(\underline{140}-\underline{143})$. Interestingly, a lack of phenotypes is often associated with genetic mutations in single effectors, and intracellular growth is completely abolished only when the Dot/Icm T4SS is inactivated. This observation, associated with the presence of multiple paralogs of the same protein, led to the concept of effector redundancy, which suggests there are compensatory roles for two proteins or set of proteins with the same biological activity or different activities that have an impact on the same pathway or cellular process. Transposon site hybridization was used to identify such so-called redundant proteins and allowed for the suggestion that there were several functional groups of effectors that concomitantly act on the same cellular pathway; consequently, their combined deletion altered L. pneumophila growth in host cells (144). Some examples are the many effectors that affect Rab1 activity or the Lgt family - Lgt1, Lgt2, and Lgt3 - that are differentially regulated during bacterial growth and affect eukaryotic protein synthesis $(\underline{145})$.

The L. pneumophila effector repertoire contains proteins that regulate the function of other bacterial effectors within the host cell, called metaeffectors. The first metaeffector described was the tandem U-box protein LubX, which ubiquitinates the host kinase Clk1 (123), and also exploits the host proteasome to temporally regulate SidH activity in the host cell (146). Since metaeffectors were first described, several others have been identified, such as SidJ, which modulates the function of SidE family proteins (147), Lpg2505, which inhibits SidI toxicity (148), and Lpg2149, which inhibits both MavC and MvcA (149). Recently, a systematic analysis 
of effector-effector regulation identified 14 additional metaeffectors whose functions can now be studied in detail $(\underline{150})$.

One of the most intriguing features of the Legionella T4SS effectors, first identified during $L$. pneumophila genome sequencing analysis, is the presence of a large variety and high number of so-called eukaryotic-like proteins and eukaryotic domain-encoding proteins ( $\underline{151})$. This finding led to the hypothesis that L. pneumophila has acquired these proteins by horizontal gene transfer from its eukaryotic hosts (amoebae) and now uses them to subvert host functions (151). Indeed, further evolutionary analyses supported this hypothesis (152-154). One of the most evident examples is the sphingosine-1-phosphate lyase-encoding gene, which different evolutionary analyses have suggested was acquired from amoeba $(\underline{155}, \underline{156})$. Furthermore, the protein encoded by this gene was shown to have the same activity as its eukaryotic counterpart, modulating the sphingolipid metabolism, and it is thus an excellent example of molecular mimicry, a main virulence strategy employed by Legionella $(\underline{109}, \underline{157})$.

Interestingly, it is not only L. pneumophila but also all Legionella species that encode remarkably large effector repertoires, as the genus harbors more than 18,000 effectors that differ surprisingly among species $(\underline{59}, \underline{158})$. Figure 3 shows the distribution of the effectors discussed in this review (see also Table 1), clearly revealing that many of them are conserved only in $L$. pneumophila and rarely present in other Legionella species. All Legionella species show evidence of long-lasting coevolution with their protozoan hosts, as the analyses of the genus genome identified effector proteins encoding 137 different eukaryotic-like domains and more than 200 eukaryotic-like proteins (모). An interesting example constitutes the group of Rab-like proteins, which are uniquely present in the effector repertoire of certain Legionella species, including L. longbeachae, that clearly have been acquired from eukaryotic organisms, probably protists, as seen in the two examples in Figure 4 (59). Like many bacterial pathogens, $L$. pneumophila also targets host Rab GTPases, for example, by recruiting Rab1 to the LCV to finally control vesicle trafficking from ER exit sites (159). The identification of bacterial Rablike GTPases in the Legionella genome suggests that these bacteria are able to subvert host cell trafficking by secreting their own Rab proteins into the host cell, and these could interact or compete with certain host Rabs during infection.

\section{<COMP: PLEASE INSERT FIGURE 3 AND 4 HERE>}


Figure 3 Distribution of 50 selected effectors from Legionella pneumophila in 58 different Legionella species and 80 Legionella strains. The sequence of the effector of L. pneumophila strain Philadelphia was used as reference to construct the tables of orthologs to define their presence or absence in 80 Legionella strains previously analyzed (9ㅗ). Blue-filled squares indicate the presence of the gene in the corresponding species based on predictions using PanOCT (the Pan-genome Ortholog Clustering Tool) with an identity cutoff of $30 \%$, a BLAST (Basic Local Alignment Search Tool) Expect (E)-value cutoff of $10^{-5}$, and a minimum percentage match length of subject and query of $65 \%$. Blue-outlined squares indicate that an orthologous gene in the corresponding species is present, but the identity and/or the minimum percentage match length is under the cutoff selected for PanOCT. Empty spaces indicate that no orthologous gene was identified in the corresponding strain. The scale bar represents 0.1 amino acid substitutions per site.

Figure 4 Phylogenetic trees of two Rab domain-containing proteins identified in the genus Legionella. (Green indicates eukaryotes; blue indicates Legionella species.) Blastp (proteinprotein BLAST; Basic Local Alignment Search Tool) was used to search for homologs of these two proteins. Maximum likelihood was used for phylogenetic reconstruction. Local support values are represented by numbers on the corresponding branches. (a) L. gratiana protein Lgra3435 was used to recruit homologs. The scale bar represents 1 amino acid substitution per site. (b) L. quateirensis protein Lqua0234 was used to recruit homologs. The scale bar represents 0.1 amino acid substitutions per site. Figure adapted from Reference $\underline{59}$.

Despite our increased knowledge about the function of the effectors secreted by $L$. pneumophila, little is known about the effectors of other Legionella species. When considering L. longbeachae, the second most frequent cause of Legionnaires' disease, more than $66 \%$ of the reported L. pneumophila Dot/Icm T4SS effectors are missing in this species, while 51 novel substrates have been identified (52). To date, only one L. longbeachae effector protein has been characterized. It was shown that SidC, similar to its homolog in L. pneumophila, is a PtdIns(4)Pbinding protein that resides on the LCV and promotes ER recruitment (160). Previous reports suggested that trafficking of the L. longbeachae vacuole might be different from that of $L$. pneumophila because the L. longbeachae LCV may acquire early and late endosomal markers (53). However, a recent report suggests that both species may develop similar replicative niches, albeit through different mechanisms, probably correlated with the specific set of effectors each species secretes into the host cell (161). Therefore, gaining better knowledge about the effectors secreted by L. longbeachae should enrich our understanding of the diverse mechanisms Legionella species utilize to successfully infect their hosts. 


\section{LEGIONELLA-AMOEBAE INTERACTIONS: A NICHE FOR THE EMERGENCE OF HUMAN PATHOGENS}

In the environment, Legionella replication within protozoa is likely the most common mechanism of bacterial proliferation $(\underline{162})$. Free-living amoebae are a group of protozoa ubiquitously found in soil and natural or man-made aquatic environments. They feed on microorganisms; and interactions over millions of years gave rise to the ability of Legionella to overcome intracellular degradation and instead survive or even replicate inside protozoa. Thus, free-living amoebae can act as Trojan horses, delivering microorganisms to new habitats and hosts in the form of intact amoeba or expelled vesicles, while protecting the microorganisms from hostile environmental conditions ( $\underline{163})$. Indeed, many medically important environmental bacteria, viruses, and fungi are associated with and are able to survive inside amoebae (164). Legionella-amoebae interactions were characterized shortly after Legionella bacteria were identified (7), and since then, the similarities between the infection of amoebae and of human macrophages have become more evident $(\underline{8}, \underline{165})$. Indeed, bacterial inactivation mechanisms are the same in amoebae and macrophages, as both consist of lysosomal degradation of the phagocytized material. Additionally, both functional outcomes (digestion and immunity, respectively) are related, as it has been proposed that they share a common evolutionary origin in metazoans $(\underline{166})$.

Among others, amoebae have been shown to be associated with bacteria from the genera Legionella, Mycobacterium, Listeria, and Chlamydia; soil fungi such as Cryptococcus species; and giant viruses belonging to the families Mimiviridae and Marseilleviridae (167). Pathogens that become specialized to infect hosts generally undergo genome reduction; however, this phenomenon is not observed in free-living amoebae-resistant bacteria (168). In contrast, it seems that Legionella bacteria undergo continuous genome expansion, with more gene gain events than losses, which is a consequence of gene acquisition by horizontal gene transfer, corroborated by the fact that the ancestral genomes were probably smaller ( $\underline{59})$.

Free-living amoebae seem to be melting pots of evolution in which giant viruses and bacteria can reside simultaneously, leading to gene fluxes in multiple directions and contributing to a socalled global mobilome $(\underline{156}, \underline{168})$. One example is a protein identified in L. pneumophila that has homologs only in the Acanthamoeba polyphaga mimivirus, indicating gene exchange involving eukaryotic viruses (154). Further examples involve other amoeba-associated bacteria, 
such as Rickettsia (169) and Amoebophilus asiaticus (170), in which recent genome sequence analyses have identified eukaryotic-like proteins in considerable numbers, similar to the Legionella species genomes. However, one of the enigmas of this genetic interchange remains to be resolved: What is the mechanism by which bacteria acquire and integrate the eukaryotic genes into their genome? One plausible explanation could be that the genetic transfer is related to RNA, which is subsequently retrotranscribed with the help of a Legionella-encoded reverse transcriptase. This would explain why no introns are present in the Legionella genes (156). Once integrated, these genes need to evolve to become specific, secreted effector proteins. Further, it has been proposed that a leaky delivery of these so-called proto-effectors to the host could allow for the selection of mutations to fine-tune protein function and to subsequently allow for the selection of an efficient C-terminal translocation signal (171).

Taken together, amoebae represent a niche allowing for the emergence of human pathogens. Thus, increased knowledge about Legionella-amoebae interactions is necessary to enable the development of new mechanisms for disease control and prevention.

\section{NEW TECHNOLOGIES AND FUTURE DIRECTIONS}

Since the identification of Legionella 40 years ago, the study of its biology has uncovered a vast arsenal of molecular tools that these bacteria use to modulate host pathways, and it has also provided insight into previously unknown mechanisms in eukaryotic cells. An example is RomA, a T4SS effector of L. pneumophila that methylates Lys14 of histone H3, a modification previously not known in mammalian cells $(\underline{121})$. The recent finding that this epigenetic modification also occurs naturally in eukaryotic cells (172) highlights how advances in research achieved by studying mechanisms of bacterial infection can be valuable to further our understanding of basic cellular processes. The development of many new techniques in recent years has allowed for more detailed studies of eukaryotic processes and Legionella-induced alterations of host functions. We envisage that future epigenetic research will embrace genomewide analyses of all known histone modifications during infection, and these will be combined with innovative genome-wide tools to perform precise epigenomic profiling, such as Internal Standard Calibrated Chromatin Immunoprecipitation, or ICeChIP (173). New tools will also ensure that the regulation of host microRNA during infection can be studied (174), as well as nucleosome positioning (175) in Legionella-infected cells. The use of Seahorse technology 
allowed to simultaneously analyze oxidative phosphorylation and glycolysis in human primary macrophages infected with L. pneumophila, which showed that specific T4SS-dependent metabolic shifts occur leading to metabolic reprogramming of the host cell (104). Future research using state-of-the-art methods - such as isotopolog profiling (176); integrated, stepwise, mass-isotopomeric flux analyses of the tricarboxylic acid cycle (177); or deep ${ }^{13} \mathrm{C}$ labeling (178) - will allow elucidation of detailed reprogramming of metabolic fluxes during infection.

For a long time, the lungs were thought to be sterile organs, but new sequencing technologies have shown that they harbor their own microbiome, like other body sites (179). Thus, nextgeneration sequencing of bronchoalveolar lavage fluids, sputum, and other clinical lung samples will allow sequencing analyses of the lung microbiome during Legionella infection ( $\underline{180})$. During disease development, Legionella might displace lung bacteria, similar to what is observed for the gut mucosa microbiome $(\underline{179}, \underline{181})$. Notably, the results obtained from such studies will be relevant for the development of new strategies for disease diagnosis, prevention, and control and possibly for the development of new therapeutics.

The expansion of new technologies together with an increased interest in understanding cell biology have contributed to the elucidation of many previously unknown cellular processes, such as exosomes production and cargo loading (182), the formation of phase-separated liquid droplets in the nucleus and cytoplasm (183), the formation of membrane nanotubes connecting cells $(\underline{184}, \underline{185})$, the repertoire of interorganelle communication $(\underline{186})$, and the mechanisms of cellular detoxification (187), including those of peroxisomes (188). Following these discoveries, questions arise, such as, what happens with these mechanisms during infection, and is Legionella manipulating these cellular processes?

Despite an increasing understanding of the biology and pathogenicity of L. pneumophila , there is still a lack of knowledge of the mechanisms of infection of L. longbeachae. The prediction of specific effector proteins in this species suggests that L. longbeachae is able to manipulate host cell pathways by means different from those used by L. pneumophila ( $\underline{52})$. The high incidence of L. longbeachae in Australia and New Zealand has been attributed to the presence of this bacterium in potting soils, which in these areas, in comparison to Europe, are mostly made from composted pine bark or sawdust. This suggests that L. longbeachae could be associated with trees and plants and that active multiplication of bacteria occurs during the composting process (189). The analysis of the L. longbeachae genome revealed that this species 
encodes for a set of enzymes probably devoted to the degradation of plant cell-wall components to be used as energy sources ( $\underline{52})$, thus supporting the hypothesis that L. longbeachae may also be associated with or infecting plants $(\underline{189})$. This finding raises the question of whether organisms other than protozoa may also be hosts of different Legionella species. Indeed, $L$. pneumophila subverts well-established immune pathways in macrophages that are not conserved in amoebae, such as caspase-mediated apoptosis or the NF- $\mathrm{BB}$ pathway; thus, it is tempting to speculate that interactions between L. pneumophila and other susceptible hosts closer to higher eukaryotes were also relevant in shaping the repertoire of effectors of this bacterium (ㅁ). Some reports support this hypothesis, as it was shown that L. pneumophila can colonize and persist within the digestive tract of the nematode Caenorhabditis elegans (190); it can cause natural pneumonia in cattle (191); and it was also identified in the microbial community of the gastrointestinal tract in Panaque nigrolineatus, a tropical herbivorous freshwater fish (192). The future discovery of Legionella hosts other than protozoa will extend our knowledge and will open up new avenues for research into Legionella-host interactions.

Taken together, four decades of research on Legionella biology and Legionnaires' disease have brought important insights into the infection strategies and the mechanisms that these intracellular pathogens use to infect their hosts and to cause disease in humans. Despite these major advances, many open questions remain. Thus, the study of the intriguing ways that Legionella bacteria are exploiting their many hosts and signaling pathways is very exciting. Without doubt, it will teach us not only about the infection strategies of the bacteria but also about eukaryotic biology, thus this will continue to be a terrific, scientific journey worth taking.

\section{DISCLOSURE STATEMENT}

The authors are not aware of any affiliations, memberships, funding, or financial holdings that might be perceived as affecting the objectivity of this review.

\section{ACKNOWLEDGMENTS}

Work in the C.B. laboratory is financed by the Institut Pasteur and funding has been received from the French Government (grants ANR-10-LABX-62-IBEID and ANR-15-CE17-0014-03 to C.B. and grant ANR-18-CE15-0005-01 to M.R.) and the "Fondation de la Recherche Médicale" 
(grant EQU201903007847 to C.B.). S.S. is a scholar in the Pasteur-Paris University (PPU)

International Ph.D. program and received a stipend from the Institut Pasteur.

\section{LITERATURE CITED}

1. Fraser DW, Tsai TR, Orenstein W, Parkin WE, Beecham HJ, et al. 1977. Legionnaires' disease: description of an epidemic of pneumonia. N. Engl. J. Med. 297(22):1189-97

2. McDade JE, Shepard CC, Fraser DW, Tsai TR, Redus MA, Dowdle WR. 1977. Legionnaires' disease: isolation of a bacterium and demonstration of its role in other respiratory disease. $N$. Engl. J. Med. 297(22):1197-203

3. Brenner DJ, Steigerwalt AG, McDade JE. 1979. Classification of the Legionnaires' disease bacterium: Legionella pneumophila, genus novum, species nova, of the family Legionellaceae, familia nova. Ann. Intern. Med. 90(4):656-58

4. McDade JE, Brenner DJ, Bozeman FM. 1979. Legionnaires' disease bacterium isolated in 1947. Ann. Intern. Med. 90(4):659-61

5. Glick TH, Gregg MB, Berman B, Mallison G, Rhodes WW, Kassanoff I. 1978. Pontiac fever: an epidemic of unknown etiology in a health department: I. Clinical and epidemiologic aspects. Am. J. Epidemiol. 107(2):149-60

6. Newton HJ, Ang DKY, van Driel IR, Hartland EL. 2010. Molecular pathogenesis of infections caused by Legionella pneumophila. Clin. Microbiol. Rev. 23(2):274-98

7. Rowbotham TJ. 1980. Preliminary report on the pathogenicity of Legionella pneumophila for freshwater and soil amoebae. J. Clin. Pathol. 33(12):1179-83

8. Escoll P, Rolando M, Gomez-Valero L, Buchrieser C. 2013. From amoeba to macrophages: exploring the molecular mechanisms of Legionella pneumophila infection in both hosts. In Molecular Mechanisms in Legionella Pathogenesis, ed. H Hilbi, pp. 1-34. Berlin: Springer

9. Taylor M, Ross K, Bentham R. 2009. Legionella, protozoa, and biofilms: interactions within complex microbial systems. Microb. Ecol. 58(3):538-47

10. Blatt SP, Parkinson MD, Pace E, Hoffman P, Dolan D, et al. 1993. Nosocomial Legionnaires' disease: aspiration as a primary mode of disease acquisition. Am. J. Med. 95(1):16-22

11. Correia AM, Ferreira JS, Borges V, Nunes A, Gomes B, et al. 2016. Probable person-to- 
person transmission of Legionnaires' disease. N. Engl. J. Med. 374(5):497-98

12. Cunha CB, Cunha BA. 2017. Legionnaire's disease since Philadelphia: lessons learned and continued progress. Infect. Dis. Clin. North Am. 31(1):1-5

13. Walker JT. 2018. The influence of climate change on waterborne disease and Legionella: a review. Perspect. Public Health 138(5):282-86

14. Beauté J. 2017. Legionnaires' disease in Europe, 2011 to 2015. Euro Surveill. 22(27):506

15. ECDC (Eur. Cent. Dis. Prev. Control). 2018. Legionnaires' disease. In ECDC: Annual Epidemiological Report for 2016. Stockholm: ECDC.

16. Shah P, Barskey A, Binder A, Edens C, Lee S, et al. 2018. Legionnaires' Disease Surveillance Summary Report, United States: 2014-2015. Atlanta, GA: CDC (Cent. Dis. Control Prev.)

17. Yu VL, Plouffe JF, Pastoris MC, Stout JE, Schousboe M, et al. 2002. Distribution of Legionella species and serogroups isolated by culture in patients with sporadic communityacquired legionellosis: an international collaborative survey. J. Infect. Dis. 186(1):127-28

18. David S, Rusniok C, Mentasti M, Gomez-Valero L, Harris SR, et al. 2016. Multiple major disease-associated clones of Legionella pneumophila have emerged recently and independently. Genome Res. 26(11):1555-64

19. Bacigalupe R, Lindsay D, Edwards G, Fitzgerald JR. 2017. Population genomics of Legionella longbeachae and hidden complexities of infection source attribution. Emerg. Infect. Dis. 23(5):750-57

20. Currie SL, Beattie TK. 2015. Compost and Legionella longbeachae: an emerging infection? Perspect. Public Health 135(6):309-15

21. Cunha BA. 2010. Legionnaires' disease: clinical differentiation from typical and other atypical pneumonias. Infect. Dis. Clin. North Am. 24(1):73-105

22. Mercante JW, Winchell JM. 2015. Current and emerging Legionella diagnostics for laboratory and outbreak investigations. Clin. Microbiol. Rev. 28(1):95-133

23. Dunne WM, Picot N, van Belkum A. 2017. Laboratory tests for Legionnaire's disease. Infect. Dis. Clin. North Am. 31(1):167-78

24. Fields BS, Benson RF, Besser RE. 2002. Legionella and Legionnaires' disease: 25 years of investigation. Clin. Microbiol. Rev. 15(3):506-26

25. Mérault N, Rusniok C, Jarraud S, Gomez-Valero L, Cazalet C, et al. 2011. Specific real-time 
PCR for simultaneous detection and identification of Legionella pneumophila serogroup 1 in water and clinical samples. Appl. Environ. Microbiol. 77(5):1708-17

26. Mentasti M, Cassier P, David S, Ginevra C, Gomez-Valero L, et al. 2017. Rapid detection and evolutionary analysis of Legionella pneumophila serogroup 1 sequence type 47. Clin. Microbiol. Infect. 23(4):264.e1-e9

27. Murdoch DR, Podmore RG, Anderson TP, Barratt K, Maze MJ, et al. 2013. Impact of routine systematic polymerase chain reaction testing on case finding for Legionnaires' disease: a pre-post comparison study. Clin. Infect. Dis. 57(9):1275-81

28. Cross KE, Mercante JW, Benitez AJ, Brown EW, Diaz MH, Winchell JM. 2016. Simultaneous detection of Legionella species and L. anisa, L. bozemanii, L. longbeachae and L. micdadei using conserved primers and multiple probes in a multiplex real-time PCR assay. Diagn. Microbiol. Infect. Dis. 85(3):295-301

29. Botelho-Nevers E, Grattard F, Viallon A, Allegra S, Jarraud S, et al. 2016. Prospective evaluation of RT-PCR on sputum versus culture, urinary antigens and serology for Legionnaire's disease diagnosis. J. Infect. 73(2):123-28

30. Bruin JP, Koshkolda T, IJzerman EPF, Lück C, Diederen BMW, et al. 2014. Isolation of ciprofloxacin-resistant Legionella pneumophila in a patient with severe pneumonia. $J$. Antimicrob. Chemother. 69(10):2869-71

31. Shadoud L, Almahmoud I, Jarraud S, Etienne J, Larrat S, et al. 2015. Hidden selection of bacterial resistance to fluoroquinolones in vivo: the case of Legionella pneumophila and humans. EBioMedicine 2(9):1179-85

32. Hennebique A, Bidart M, Jarraud S, Beraud L, Schwebel C, et al. 2017. Digital PCR for detection and quantification of fluoroquinolone resistance in Legionella pneumophila. Antimicrob. Agents Chemother. 61(9):794

33. Pedro-Botet L, Yu VL. 2006. Legionella: macrolides or quinolones? Clin. Microbiol. Infect. 12(Suppl. 3):25-30

34. Potts A, Donaghy M, Marley M, Othieno R, Stevenson J, et al. 2013. Cluster of Legionnaires' disease cases caused by Legionella longbeachae serogroup 1, Scotland, August to September 2013. Euro Surveill. 18(50):20656

35. de Bruin L, Timmerman CP, Huisman PM, Heidt J. 2018. Legionella longbeachae: Don't miss it! Neth J. Med. 76(6):294-97 
36. MMWR (Morbid. Mortal. Wkly. Rep.). 2000. Legionnaires' disease associated with potting soil-California, Oregon, and Washington, May-June 2000. MMWR 49(34):777-78

37. Picard-Masson M, Lajoie É, Lord J, Lalancette C, Marchand G, et al. 2016. Two related occupational cases of Legionella longbeachae infection, Quebec, Canada. Emerg. Infect. Dis. 22(7):1289-91

38. Phares CR, Wangroongsarb P, Chantra S, Paveenkitiporn W, Tondella M-L, et al. 2007. Epidemiology of severe pneumonia caused by Legionella longbeachae, Mycoplasma pneumoniae, and Chlamydia pneumoniae: 1-year, population-based surveillance for severe pneumonia in Thailand. Clin. Infect. Dis. 45(12):e147-55

39. Wei S-H, Tseng L-R, Tan J-K, Cheng C-Y, Hsu Y-T, et al. 2014. Legionnaires' disease caused by Legionella longbeachae in Taiwan, 2006-2010. Int. J. Infect. Dis. 19:95-97

40. Cameron RL, Pollock KGJ, Lindsay DSJ, Anderson E. 2016. Comparison of Legionella longbeachae and Legionella pneumophila cases in Scotland; implications for diagnosis, treatment and public health response. J. Med. Microbiol. 65(2):142-46

41. Kenagy E, Priest PC, Cameron CM, Smith D, Scott P, et al. 2017. Risk factors for Legionella longbeachae Legionnaires' disease, New Zealand. Emerg. Infect. Dis. 23(7):1148-54

42. Thornley CN, Harte DJ, Weir RP, Allen LJ, Knightbridge KJ, Wood PRT. 2017. Legionella longbeachae detected in an industrial cooling tower linked to a legionellosis outbreak, New Zealand, 2015: possible waterborne transmission? Epidemiol. Infect. 145(11):2382-89

43. Molofsky AB, Swanson MS. 2004. Differentiate to thrive: lessons from the Legionella pneumophila life cycle. Mol. Microbiol. 53(1):29-40

44. Oliva G, Sahr T, Buchrieser C. 2018. The life cycle of Legionella pneumophila: Cellular differentiation is linked to virulence and metabolism. Front. Cell. Infect. Microbiol. 8:3

45. Brüggemann H, Hagman A, Jules M, Sismeiro O, Dillies M-A, et al. 2006. Virulence strategies for infecting phagocytes deduced from the in vivo transcriptional program of Legionella pneumophila. Cell. Microbiol. 8(8):1228-40

46. Byrne B, Swanson MS. 1998. Expression of Legionella pneumophila virulence traits in response to growth conditions. Infect. Immun. 66(7):3029-34

47. Molofsky AB, Swanson MS. 2003. Legionella pneumophila CsrA is a pivotal repressor of transmission traits and activator of replication. Mol. Microbiol. 50(2):445-61

48. Fettes PS, Forsbach-Birk V, Lynch D, Marre R. 2001. Overexpresssion of a Legionella 
pneumophila homologue of the Escherichia coli regulator $\operatorname{csr} A$ affects cell size, flagellation, and pigmentation. Int. J. Med. Microbiol. 291(5):353-60

49. Rasis M, Segal G. 2009. The LetA-RsmYZ-CsrA regulatory cascade, together with RpoS and PmrA, post-transcriptionally regulates stationary phase activation of Legionella pneumophila Icm/Dot effectors. Mol. Microbiol. 72(4):995-1010

50. Sahr T, Rusniok C, Dervins-Ravault D, Sismeiro O, Coppée J-Y, Buchrieser C. 2012. Deep sequencing defines the transcriptional map of Legionella pneumophila and identifies growth phase-dependent regulated ncRNAs implicated in virulence. RNA Biol. 9(4):503-19

51. Sahr T, Rusniok C, Impens F, Oliva G, Sismeiro O, et al. 2017. The Legionella pneumophila genome evolved to accommodate multiple regulatory mechanisms controlled by the CsrAsystem. PLOS Genet. 13(2):e1006629

52. Cazalet C, Gomez-Valero L, Rusniok C, Lomma M, Dervins-Ravault D, et al. 2010. Analysis of the Legionella longbeachae genome and transcriptome uncovers unique strategies to cause Legionnaires' disease. PLOS Genet. 6(2):e1000851

53. Asare R, Abu Kwaik Y. 2007. Early trafficking and intracellular replication of Legionella longbeachae within an ER-derived late endosome-like phagosome. Cell. Microbiol. 9(6):1571-87

54. Kozak NA, Buss M, Lucas CE, Frace M, Govil D, et al. 2010. Virulence factors encoded by Legionella longbeachae identified on the basis of the genome sequence analysis of clinical isolate D-4968. J. Bacteriol. 192(4):1030-44

55. Gomez-Valero L, Rusniok C, Cazalet C, Buchrieser C. 2011. Comparative and functional genomics of Legionella identified eukaryotic like proteins as key players in host-pathogen interactions. Front. Microbiol. 2:208

56. Ren T, Zamboni DS, Roy CR, Dietrich WF, Vance RE. 2006. Flagellin-deficient Legionella mutants evade caspase-1- and Naip 5-mediated macrophage immunity. PLOS Pathog. 2(3):e18

57. Massis LM, Assis-Marques MA, Castanheira FVS, Capobianco YJ, Balestra AC, et al. 2017. Legionella longbeachae is immunologically silent and highly virulent in vivo. J. Infect. Dis. 215(3):440-51

58. Qin T, Zhou H, Ren H, Liu W. 2017. Distribution of secretion systems in the genus Legionella and its correlation with pathogenicity. Front. Microbiol. 8:388 
59. Gomez-Valero L, Rusniok C, Carson D, Mondino S, Pérez-Cobas AE, et al. 2019. More than 18,000 effectors in the Legionella genus genome provide multiple, independent combinations for replication in human cells. PNAS 116(6):2265-73

60. Cianciotto NP. 2005. Type II secretion: a protein secretion system for all seasons. Trends Microbiol. 13(12):581-88

61. Truchan HK, Christman HD, White RC, Rutledge NS, Cianciotto NP. 2017. Type II secretion substrates of Legionella pneumophila translocate out of the pathogen-occupied vacuole via a semipermeable membrane. mBio 8(3):4797

62. Cianciotto NP. 2009. Many substrates and functions of type II secretion: lessons learned from Legionella pneumophila. Future Microbiol. 4(7):797-805

63. Cianciotto NP. 2013. Type II secretion and Legionella virulence. In Molecular Mechanisms in Legionella Pathogenesis, ed. H Hilbi, pp. 81-102. Berlin: Springer

64. DebRoy S, Dao J, Söderberg M, Rossier O, Cianciotto NP. 2006. Legionella pneumophila type II secretome reveals unique exoproteins and a chitinase that promotes bacterial persistence in the lung. PNAS 103(50):19146-51

65. Berger KH, Isberg RR. 1993. Two distinct defects in intracellular growth complemented by a single genetic locus in Legionella pneumophila. Mol. Microbiol. 7(1):7-19

66. Marra A, Blander SJ, Horwitz MA, Shuman HA. 1992. Identification of a Legionella pneumophila locus required for intracellular multiplication in human macrophages. PNAS 89(20):9607-11

67. Jeong KC, Ghosal D, Chang Y-W, Jensen GJ, Vogel JP. 2017. Polar delivery of Legionella type IV secretion system substrates is essential for virulence. PNAS 114(30):8077-82

68. Charpentier X, Gabay JE, Reyes M, Zhu JW, Weiss A, Shuman HA. 2009. Chemical genetics reveals bacterial and host cell functions critical for type IV effector translocation by Legionella pneumophila. PLOS Pathog. 5(7):e1000501

69. Hilbi H, Segal G, Shuman HA. 2001. Icm/Dot-dependent upregulation of phagocytosis by Legionella pneumophila. Mol. Microbiol. 42(3):603-17

70. Watarai M, Derre I, Kirby J, Growney JD, Dietrich WF, Isberg RR. 2001. Legionella pneumophila is internalized by a macropinocytotic uptake pathway controlled by the Dot/Icm system and the mouse Lgn1 locus. J. Exp. Med. 194(8):1081-96

71. Weber S, Wagner M, Hilbi H. 2014. Live-cell imaging of phosphoinositide dynamics and 
membrane architecture during Legionella infection. mBio 5(1):e00839-13

72. Fuche F, Vianney A, Andrea C, Doublet P, Gilbert C. 2015. Functional type 1 secretion system involved in Legionella pneumophila virulence. J. Bacteriol. 197(3):563-71

73. Horwitz MA. 1983. The Legionnaires' disease bacterium (Legionella pneumophila) inhibits phagosome-lysosome fusion in human monocytes. J. Exp. Med. 158(6):2108-26

74. Xu L, Shen X, Bryan A, Banga S, Swanson MS, Luo Z-Q. 2010. Inhibition of host vacuolar $\mathrm{H}^{+}$-ATPase activity by a Legionella pneumophila effector. PLOS Pathog. 6(3):e1000822

75. Prevost MS, Pinotsis N, Dumoux M, Hayward RD, Waksman G. 2017. The Legionella effector WipB is a translocated Ser/Thr phosphatase that targets the host lysosomal nutrient sensing machinery. Sci. Rep. 7(1):9450

76. Urwyler S, Nyfeler Y, Ragaz C, Lee H, Mueller LN, et al. 2009. Proteome analysis of Legionella vacuoles purified by magnetic immunoseparation reveals secretory and endosomal GTPases. Traffic 10(1):76-87

77. Hoffmann C, Finsel I, Otto A, Pfaffinger G, Rothmeier E, et al. 2014. Functional analysis of novel Rab GTPases identified in the proteome of purified Legionella-containing vacuoles from macrophages. Cell. Microbiol. 16(7):1034-52

78. Lawe DC, Chawla A, Merithew E, Dumas J, Carrington W, et al. 2002. Sequential roles for phosphatidylinositol 3-phosphate and Rab5 in tethering and fusion of early endosomes via their interaction with EEA1. J. Biol. Chem. 277(10):8611-17

79. Gaspar AH, Machner MP. 2014. VipD is a Rab5-activated phospholipase A1 that protects Legionella pneumophila from endosomal fusion. PNAS 111(12):4560-65

80. Mousnier A, Schroeder GN, Stoneham CA, So EC, Garnett JA, et al. 2014. A new method to determine in vivo interactomes reveals binding of the Legionella pneumophila effector PieE to multiple Rab GTPases. mBio 5(4):e01148-14

81. Rink J, Ghigo E, Kalaidzidis Y, Zerial M. 2005. Rab conversion as a mechanism of progression from early to late endosomes. Cell 122(5):735-49

82. Rojas R, van Vlijmen T, Mardones GA, Prabhu Y, Rojas AL, et al. 2008. Regulation of retromer recruitment to endosomes by sequential action of Rab5 and Rab7. J. Cell Biol. 183(3):513-26

83. Finsel I, Ragaz C, Hoffmann C, Harrison CF, Weber S, et al. 2013. The Legionella effector RidL inhibits retrograde trafficking to promote intracellular replication. Cell Host Microbe 
14(1):38-50

84. Robinson CG, Roy CR. 2006. Attachment and fusion of endoplasmic reticulum with vacuoles containing Legionella pneumophila. Cell. Microbiol. 8(5):793-805

85. Kagan JC, Roy CR. 2002. Legionella phagosomes intercept vesicular traffic from endoplasmic reticulum exit sites. Nat. Cell Biol. 4(12):945-54

86. Schoebel S, Oesterlin LK, Blankenfeldt W, Goody RS, Itzen A. 2009. RabGDI displacement by DrrA from Legionella is a consequence of its guanine nucleotide exchange activity. Mol. Cell 36(6):1060-72

87. Murata T, Delprato A, Ingmundson A, Toomre DK, Lambright DG, Roy CR. 2006. The Legionella pneumophila effector protein DrrA is a Rab1 guanine nucleotide-exchange factor. Nat. Cell Biol. 8(9):971-77

88. Machner MP, Isberg RR. 2007. A bifunctional bacterial protein links GDI displacement to Rab1 activation. Science 318(5852):974-77

89. Müller MP, Peters H, Blümer J, Blankenfeldt W, Goody RS, Itzen A. 2010. The Legionella effector protein DrrA AMPylates the membrane traffic regulator Rab1b. Science 329(5994):946-49

90. Machner MP, Isberg RR. 2006. Targeting of host Rab GTPase function by the intravacuolar pathogen Legionella pneumophila. Dev. Cell 11(1):47-56

91. Neunuebel MR, Chen Y, Gaspar AH, Backlund PS, Yergey A, Machner MP. 2011. DeAMPylation of the small GTPase Rab1 by the pathogen Legionella pneumophila. Science 333(6041):453-56

92. Ingmundson A, Delprato A, Lambright DG, Roy CR. 2007. Legionella pneumophila proteins that regulate Rab1 membrane cycling. Nature 450(7168):365-69

93. Mukherjee S, Liu X, Arasaki K, McDonough J, Galán JE, Roy CR. 2011. Modulation of Rab GTPase function by a protein phosphocholine transferase. Nature 477(7362):103-6

94. Tan Y, Arnold RJ, Luo Z-Q. 2011. Legionella pneumophila regulates the small GTPase Rab1 activity by reversible phosphorylcholination. PNAS 108(52):21212-17

95. Nagai H, Kagan JC, Zhu X, Kahn RA, Roy CR. 2002. A bacterial guanine nucleotide exchange factor activates ARF on Legionella phagosomes. Science 295(5555):679-82

96. Hubber A, Arasaki K, Nakatsu F, Hardiman C, Lambright D, et al. 2014. The machinery at endoplasmic reticulum-plasma membrane contact sites contributes to spatial regulation of 
multiple Legionella effector proteins. PLOS Pathog. 10(7):e1004222

97. Haenssler E, Ramabhadran V, Murphy CS, Heidtman MI, Isberg RR. 2015. Endoplasmic reticulum tubule protein reticulon 4 associates with the Legionella pneumophila vacuole and with translocated substrate Ceg9. Infect. Immun. 83(9):3479-89

98. Arasaki K, Roy CR. 2010. Legionella pneumophila promotes functional interactions between plasma membrane syntaxins and Sec22b. Traffic 11(5):587-600

99. Jahn R, Scheller RH. 2006. SNAREs—engines for membrane fusion. Nat. Rev. Mol. Cell Biol. 7(9):631-43

100. Arasaki K, Toomre DK, Roy CR. 2012. The Legionella pneumophila effector DrrA is sufficient to stimulate SNARE-dependent membrane fusion. Cell Host Microbe 11(1):46-57

101. King NP, Newton P, Schuelein R, Brown DL, Petru M, et al. 2015. Soluble NSF attachment protein receptor molecular mimicry by a Legionella pneumophila Dot/Icm effector. Cell. Microbiol. 17(6):767-84

102. Bennett TL, Kraft SM, Reaves BJ, Mima J, O’Brien KM, Starai VJ. 2013. LegC3, an effector protein from Legionella pneumophila, inhibits homotypic yeast vacuole fusion in vivo and in vitro. PLOS ONE 8(2):e56798

103. Rothmeier E, Pfaffinger G, Hoffmann C, Harrison CF, Grabmayr H, et al. 2013. Activation of Ran GTPase by a Legionella effector promotes microtubule polymerization, pathogen vacuole motility and infection. PLOS Pathog. 9(9):e1003598

104. Escoll P, Song O-R, Viana F, Steiner B, Lagache T, et al. 2017. Legionella pneumophila modulates mitochondrial dynamics to trigger metabolic repurposing of infected macrophages. Cell Host Microbe 22(3):302-7

105. Escoll P, Buchrieser C. 2018. Metabolic reprogramming of host cells upon bacterial infection: Why shift to a Warburg-like metabolism? FEBS J. 285(12):2146-60

106. Amer AO, Swanson MS. 2005. Autophagy is an immediate macrophage response to Legionella pneumophila. Cell. Microbiol. 7(6):765-78

107. Choy A, Dancourt J, Mugo B, O'Connor TJ, Isberg RR, et al. 2012. The Legionella effector RavZ inhibits host autophagy through irreversible Atg8 deconjugation. Science 338(6110):1072-76

108. Arasaki K, Mikami Y, Shames SR, Inoue H, Wakana Y, Tagaya M. 2017. Legionella effector Lpg1137 shuts down ER-mitochondria communication through cleavage of syntaxin 
17. Nat. Commun. 8:15406

109. Rolando M, Escoll P, Nora T, Botti J, Boitez V, et al. 2016. Legionella pneumophila S1Plyase targets host sphingolipid metabolism and restrains autophagy. PNAS 113(7):1901-6

110. Kaneko T, Stogios PJ, Ruan X, Voss C, Evdokimova E, et al. 2018. Identification and characterization of a large family of superbinding bacterial SH2 domains. Nat. Commun. 9(1):4549

111. Lee P-C, Machner MP. 2018. The Legionella effector kinase LegK7 hijacks the host Hippo pathway to promote infection. Cell Host Microbe 24(3):429-438.e6

112. Bartfeld S, Engels C, Bauer B, Aurass P, Flieger A, et al. 2009. Temporal resolution of twotracked NF- $\kappa$ B activation by Legionella pneumophila. Cell. Microbiol. 11(11):1638-51

113. Ge J, Xu H, Li T, Zhou Y, Zhang Z, et al. 2009. A Legionella type IV effector activates the

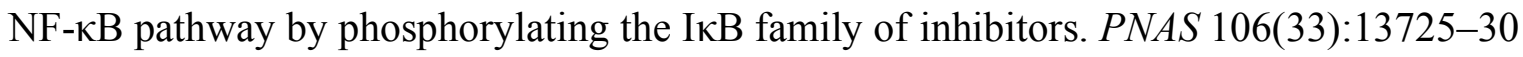

114. Losick VP, Haenssler E, Moy M-Y, Isberg RR. 2010. LnaB: a Legionella pneumophila activator of NF-кB. Cell. Microbiol. 12(8):1083-97

115. Gan N, Nakayasu ES, Hollenbeck PJ, Luo Z-Q. 2019. Legionella pneumophila inhibits immune signalling via MavC-mediated transglutaminase-induced ubiquitination of UBE2N. Nat. Microbiol. 4(1):134-43

116. Fontana MF, Banga S, Barry KC, Shen X, Tan Y, et al. 2011. Secreted bacterial effectors that inhibit host protein synthesis are critical for induction of the innate immune response to virulent Legionella pneumophila. PLOS Pathog. 7(2):e1001289

117. Welsh CT, Summersgill JT, Miller RD. 2004. Increases in c-Jun N-terminal kinase/stressactivated protein kinase and p38 activity in monocyte-derived macrophages following the uptake of Legionella pneumophila. Infect. Immun. 72(3):1512-18

118. Shin S, Case CL, Archer KA, Nogueira CV, Kobayashi KS, et al. 2008. Type IV secretiondependent activation of host MAP kinases induces an increased proinflammatory cytokine response to Legionella pneumophila. PLOS Pathog. 4(11):e1000220

119. Fontana MF, Shin S, Vance RE. 2012. Activation of host mitogen-activated protein kinases by secreted Legionella pneumophila effectors that inhibit host protein translation. Infect. Immun. 80(10):3570-75

120. Quaile AT, Stogios PJ, Egorova O, Evdokimova E, Valleau D, et al. 2018. The Legionella pneumophila effector Ceg4 is a phosphotyrosine phosphatase that attenuates activation of 
eukaryotic MAPK pathways. J. Biol. Chem. 293(9):3307-20

121. Rolando M, Sanulli S, Rusniok C, Gomez-Valero L, Bertholet C, et al. 2013. Legionella pneumophila effector RomA uniquely modifies host chromatin to repress gene expression and promote intracellular bacterial replication. Cell Host Microbe 13(4):395-405

122. Schuelein R, Spencer H, Dagley LF, Li PF, Luo L, et al. 2018. Targeting of RNA Polymerase II by a nuclear Legionella pneumophila Dot/Icm effector SnpL. Cell. Microbiol. 20(9):e12852

123. Kubori T, Hyakutake A, Nagai H. 2008. Legionella translocates an E3 ubiquitin ligase that has multiple U-boxes with distinct functions. Mol. Microbiol. 67(6):1307-19

124. Lin Y-H, Doms AG, Cheng E, Kim B, Evans TR, Machner MP. 2015. Host cell-catalyzed $S$-palmitoylation mediates Golgi targeting of the Legionella ubiquitin ligase GobX. J. Biol. Chem. 290(42):25766-81

125. Lin Y-H, Lucas M, Evans TR, Abascal-Palacios G, Doms AG, et al. 2018. RavN is a member of a previously unrecognized group of Legionella pneumophila E3 ubiquitin ligases. PLOS Pathog. 14(2):e1006897

126. Ragaz C, Pietsch H, Urwyler S, Tiaden A, Weber SS, Hilbi H. 2008. The Legionella pneumophila phosphatidylinositol-4 phosphate-binding type IV substrate SidC recruits endoplasmic reticulum vesicles to a replication-permissive vacuole. Cell. Microbiol. 10(12):2416-33

127. Hsu F, Luo X, Qiu J, Teng Y-B, Jin J, et al. 2014. The Legionella effector SidC defines a unique family of ubiquitin ligases important for bacterial phagosomal remodeling. PNAS 111(29):10538-43

128. Ensminger AW, Isberg RR. 2010. E3 ubiquitin ligase activity and targeting of BAT3 by multiple Legionella pneumophila translocated substrates. Infect. Immun. 78(9):3905-19

129. Lomma M, Dervins-Ravault D, Rolando M, Nora T, Newton HJ, et al. 2010. The Legionella pneumophila F-box protein Lpp2082 (AnkB) modulates ubiquitination of the host protein parvin B and promotes intracellular replication. Cell. Microbiol. 12(9):1272-91

130. Price CTD, Al-Quadan T, Santic M, Rosenshine I, Abu Kwaik Y. 2011. Host proteasomal degradation generates amino acids essential for intracellular bacterial growth. Science 334(6062):1553-57

131. Qiu J, Sheedlo MJ, Yu K, Tan Y, Nakayasu ES, et al. 2016. Ubiquitination independent of 
E1 and E2 enzymes by bacterial effectors. Nature 533(7601):120-24

132. Bhogaraju S, Kalayil S, Liu Y, Bonn F, Colby T, et al. 2016. Phosphoribosylation of ubiquitin promotes serine ubiquitination and impairs conventional ubiquitination. Cell 167(6):1636-1649

133. Qiu J, Yu K, Fei X, Liu Y, Nakayasu ES, et al. 2017. A unique deubiquitinase that deconjugates phosphoribosyl-linked protein ubiquitination. Cell Res. 27(7):865-81

134. Kotewicz KM, Ramabhadran V, Sjoblom N, Vogel JP, Haenssler E, et al. 2017. A single Legionella effector catalyzes a multistep ubiquitination pathway to rearrange tubular endoplasmic reticulum for replication. Cell Host Microbe 21(2):169-81

135. Kubori T, Kitao T, Ando H, Nagai H. 2018. LotA, a Legionella deubiquitinase, has dual catalytic activity and contributes to intracellular growth. Cell. Microbiol. 20(7):e12840

136. Luo Z-Q. 2011. Striking a balance: modulation of host cell death pathways by Legionella pneumophila. Front. Microbiol. 2:36

137. Banga S, Gao P, Shen X, Fiscus V, Zong W-X, et al. 2007. Legionella pneumophila inhibits macrophage apoptosis by targeting pro-death members of the Bcl 2 protein family. PNAS 104(12):5121-26

138. Laguna RK, Creasey EA, Li Z, Valtz N, Isberg RR. 2006. A Legionella pneumophilatranslocated substrate that is required for growth within macrophages and protection from host cell death. PNAS 103(49):18745-50

139. Zhu W, Hammad LA, Hsu F, Mao Y, Luo Z-Q. 2013. Induction of caspase 3 activation by multiple Legionella pneumophila Dot/Icm substrates. Cell. Microbiol. 15(11):1783-95

140. Lifshitz Z, Burstein D, Peeri M, Zusman T, Schwartz K, et al. 2013. Computational modeling and experimental validation of the Legionella and Coxiella virulence-related typeIVB secretion signal. PNAS 110(8):E707-15

141. Zhu W, Banga S, Tan Y, Zheng C, Stephenson R, et al. 2011. Comprehensive identification of protein substrates of the Dot/Icm type IV transporter of Legionella pneumophila. PLOS ONE 6(3):e17638

142. Escoll P, Mondino S, Rolando M, Buchrieser C. 2016. Targeting of host organelles by pathogenic bacteria: a sophisticated subversion strategy. Nat. Rev. Microbiol. 14(1):5-19

143. Schroeder GN. 2017. The toolbox for uncovering the functions of Legionella Dot/Icm type IVb secretion system effectors: current state and future directions. Front. Cell. Infect. 
Microbiol. 7:528

144. O’Connor TJ, Adepoju Y, Boyd D, Isberg RR. 2011. Minimization of the Legionella pneumophila genome reveals chromosomal regions involved in host range expansion. PNAS 108(36):14733-40

145. Belyi Y, Jank T, Aktories K. 2013. Cytotoxic glucosyltransferases of Legionella pneumophila. In Molecular Mechanisms in Legionella Pathogenesis, ed. H Hilbi, pp. 21126. Berlin: Springer

146. Kubori T, Shinzawa N, Kanuka H, Nagai H. 2010. Legionella metaeffector exploits host proteasome to temporally regulate cognate effector. PLOS Pathog. 6(12):e1001216

147. Jeong KC, Sexton JA, Vogel JP. 2015. Spatiotemporal regulation of a Legionella pneumophila T4SS substrate by the metaeffector SidJ. PLOS Pathog. 11(3):e1004695

148. Shames SR, Liu L, Havey JC, Schofield WB, Goodman AL, Roy CR. 2017. Multiple Legionella pneumophila effector virulence phenotypes revealed through high-throughput analysis of targeted mutant libraries. PNAS 114(48):E10446-54

149. Valleau D, Quaile AT, Cui H, Xu X, Evdokimova E, et al. 2018. Discovery of ubiquitin deamidases in the pathogenic arsenal of Legionella pneumophila. Cell Rep. 23(2):568-83

150. Urbanus ML, Quaile AT, Stogios PJ, Morar M, Rao C, et al. 2016. Diverse mechanisms of metaeffector activity in an intracellular bacterial pathogen, Legionella pneumophila. Mol. Syst. Biol. 12(12):893

151. Cazalet C, Rusniok C, Brüggemann H, Zidane N, Magnier A, et al. 2004. Evidence in the Legionella pneumophila genome for exploitation of host cell functions and high genome plasticity. Nat. Genet. 36(11):1165-73

152. de Felipe KS, Pampou S, Jovanovic OS, Pericone CD, Ye SF, et al. 2005. Evidence for acquisition of Legionella type IV secretion substrates via interdomain horizontal gene transfer. J. Bacteriol. 187(22):7716-26

153. Gomez-Valero L, Rusniok C, Buchrieser C. 2009. Legionella pneumophila: population genetics, phylogeny and genomics. Infect. Genet. Evol. 9(5):727-39

154. Lurie-Weinberger MN, Gomez-Valero L, Merault N, Glöckner G, Buchrieser C, Gophna U. 2010. The origins of eukaryotic-like proteins in Legionella pneumophila. Int. J. Med. Microbiol. 300(7):470-81

155. Degtyar E, Zusman T, Ehrlich M, Segal G. 2009. A Legionella effector acquired from 
protozoa is involved in sphingolipids metabolism and is targeted to the host cell mitochondria. Cell. Microbiol. 11(8):1219-35

156. Gomez-Valero L, Buchrieser C. 2013. Genome dynamics in Legionella: the basis of versatility and adaptation to intracellular replication. Cold Spring Harb. Perspect. Med. 3(6):a009993

157. Nora T, Lomma M, Gomez-Valero L, Buchrieser C. 2009. Molecular mimicry: an important virulence strategy employed by Legionella pneumophila to subvert host functions. Future Microbiol. 4(6):691-701

158. Burstein D, Amaro F, Zusman T, Lifshitz Z, Cohen O, et al. 2016. Genomic analysis of 38 Legionella species identifies large and diverse effector repertoires. Nat. Genet. 48(2):167-75

159. Weber MM, Faris R. 2018. Subversion of the endocytic and secretory pathways by bacterial effector proteins. Front. Cell Dev. Biol. 6:1

160. Dolinsky S, Haneburger I, Cichy A, Hannemann M, Itzen A, Hilbi H. 2014. The Legionella longbeachae Icm/Dot substrate SidC selectively binds phosphatidylinositol 4-phosphate with nanomolar affinity and promotes pathogen vacuole-endoplasmic reticulum interactions. Infect. Immun. 82(10):4021-33

161. Wood RE, Newton P, Latomanski EA, Newton HJ. 2015. Dot/Icm effector translocation by Legionella longbeachae creates a replicative vacuole similar to that of Legionella pneumophila despite translocation of distinct effector repertoires. Infect. Immun. 83(10):4081-92

162. Boamah DK, Zhou G, Ensminger AW, O'Connor TJ. 2017. From many hosts, one accidental pathogen: the diverse protozoan hosts of Legionella. Front. Cell. Infect. Microbiol. $7: 477$

163. Barker J, Brown MR. 1994. Trojan horses of the microbial world: protozoa and the survival of bacterial pathogens in the environment. Microbiology 140(6):1253-59

164. Scheid P. 2014. Relevance of free-living amoebae as hosts for phylogenetically diverse microorganisms. Parasitol. Res. 113(7):2407-14

165. Richards AM, Von Dwingelo JE, Price CT, Abu Kwaik Y. 2013. Cellular microbiology and molecular ecology of Legionella-amoeba interaction. Virulence 4(4):307-14

166. Broderick NA. 2015. A common origin for immunity and digestion. Front. Immunol. 6:72

167. Casadevall A, Fu MS, Guimaraes AJ, Albuquerque P. 2019. The 'amoeboid predator- 
fungal animal virulence' hypothesis. J. Fungi 5(1):10

168. Moliner C, Fournier P-E, Raoult D. 2010. Genome analysis of microorganisms living in amoebae reveals a melting pot of evolution. FEMS Microbiol. Rev. 34(3):281-94

169. Schulz F, Martijn J, Wascher F, Lagkouvardos I, Kostanjšek R, et al. 2016. A Rickettsiales symbiont of amoebae with ancient features. Environ. Microbiol. 18(8):2326-42

170. Schmitz-Esser S, Tischler P, Arnold R, Montanaro J, Wagner M, et al. 2010. The genome of the amoeba symbiont "Candidatus Amoebophilus asiaticus" reveals common mechanisms for host cell interaction among amoeba-associated bacteria. J. Bacteriol. 192(4):1045-57

171. Ensminger AW. 2016. Legionella pneumophila, armed to the hilt: justifying the largest arsenal of effectors in the bacterial world. Curr. Opin. Microbiol. 29:74-80

172. Zhao B, Xu W, Rong B, Chen G, Ye X, et al. 2018. H3K14me3 genomic distributions and its regulation by KDM4 family demethylases. Cell Res. 28(11):1118-20

173. Grzybowski AT, Chen Z, Ruthenburg AJ. 2015. Calibrating ChIP-Seq with nucleosomal internal standards to measure histone modification density genome wide. Mol. Cell 58(5):886-99

174. Bansal A, Singh TR, Chauhan RS. 2017. A novel miRNA analysis framework to analyze differential biological networks. Sci. Rep. 7(1):14604

175. Schones DE, Cui K, Cuddapah S, Roh T-Y, Barski A, et al. 2008. Dynamic regulation of nucleosome positioning in the human genome. Cell 132(5):887-98

176. Eylert E, Herrmann V, Jules M, Gillmaier N, Lautner M, et al. 2010. Isotopologue profiling of Legionella pneumophila: role of serine and glucose as carbon substrates. J. Biol. Chem. 285(29):22232-43

177. Alves TC, Pongratz RL, Zhao X, Yarborough O, Sereda S, et al. 2015. Integrated, stepwise, mass-isotopomeric flux analysis of the TCA cycle. Cell Metab. 22(5):936-47

178. Grankvist N, Watrous JD, Lagerborg KA, Lyutvinskiy Y, Jain M, Nilsson R. 2018. Profiling the metabolism of human cells by deep ${ }^{13} \mathrm{C}$ labelling. Cell Chem. Biol. 25(11):1419-1427

179. Dickson RP, Erb-Downward JR, Huffnagle GB. 2015. Homeostasis and its disruption in the lung microbiome. Am. J. Physiol. Lung Cell. Mol. Physiol. 309(10):L1047-55

180. Pérez-Cobas AE, Buchrieser C. 2019. Analysis of the pulmonary microbiome composition of Legionella pneumophila-infected patients. Methods Mol. Biol. 1921(4):429-43 
181. Pham TAN, Lawley TD. 2014. Emerging insights on intestinal dysbiosis during bacterial infections. Curr. Opin. Microbiol. 17:67-74

182. Hadifar S, Fateh A, Yousefi MH, Siadat SD, Vaziri F. 2019. Exosomes in tuberculosis: still terra incognita? J. Cell. Physiol. 234(3):2104-11

183. Uversky VN. 2017. Intrinsically disordered proteins in overcrowded milieu: membrane-less organelles, phase separation, and intrinsic disorder. Curr. Opin. Struct. Biol. 44:18-30

184. Onfelt B, Nedvetzki S, Benninger RKP, Purbhoo MA, Sowinski S, et al. 2006. Structurally distinct membrane nanotubes between human macrophages support long-distance vesicular traffic or surfing of bacteria. J. Immunol. 177(12):8476-83

185. Baidya AK, Bhattacharya S, Dubey GP, Mamou G, Ben-Yehuda S. 2018. Bacterial nanotubes: a conduit for intercellular molecular trade. Curr. Opin. Microbiol. 42:1-6

186. Gottschling DE, Nyström T. 2017. The upsides and downsides of organelle interconnectivity. Cell 169(1):24-34

187. Staerck C, Gastebois A, Vandeputte P, Calenda A, Larcher G, et al. 2017. Microbial antioxidant defense enzymes. Microb. Pathog. 110:56-65

188. Islinger M, Voelkl A, Fahimi HD, Schrader M. 2018. The peroxisome: an update on mysteries 2.0. Histochem. Cell Biol. 150(5):443-71

189. Whiley H, Bentham R. 2011. Legionella longbeachae and legionellosis. Emerg. Infect. Dis. 17(4):579-83

190. Brassinga AKC, Kinchen JM, Cupp ME, Day SR, Hoffman PS, Sifri CD. 2010. Caenorhabditis is a metazoan host for Legionella. Cell. Microbiol. 12(3):343-61

191. Fabbi M, Pastoris MC, Scanziani E, Magnino S, Di Matteo L. 1998. Epidemiological and environmental investigations of Legionella pneumophila infection in cattle and case report of fatal pneumonia in a calf. J. Clin. Microbiol. 36(7):1942-47

192. McDonald R, Schreier HJ, Watts JEM. 2012. Phylogenetic analysis of microbial communities in different regions of the gastrointestinal tract in Panaque nigrolineatus, a wood-eating fish. PLOS ONE 7(10):e48018

193. Schoebel S, Blankenfeldt W, Goody RS, Itzen A. 2010. High-affinity binding of phosphatidylinositol 4-phosphate by Legionella pneumophila DrrA. EMBO Rep. 11(8):598604 
Table 1 Selected secreted effectors of Legionella with functions discussed in the review

\begin{tabular}{|c|c|c|c|c|}
\hline \multirow{2}{*}{ Effector $^{\mathrm{a}}$} & \multicolumn{2}{|c|}{ Gene $^{b}$} & \multirow{2}{*}{ Cellular target and function } & \multirow{2}{*}{ Reference } \\
\hline & Paris strain & Philadelphia strain & & \\
\hline \multicolumn{5}{|c|}{ Bacterial uptake and evasion from the endocytic maturation pathway } \\
\hline SidK & $\operatorname{lpp} 1030$ & $\operatorname{lpg} 0968$ & \multirow{2}{*}{$\begin{array}{l}\text { Blocks the host vacuolar ATPase to restrain } \\
\text { vacuolar acidification }\end{array}$} & 74 \\
\hline WipB & $\operatorname{lpp} 2775$ & $\operatorname{lpg} 2718$ & & $\underline{75}$ \\
\hline VipD & lpp2888 & $\operatorname{lpg} 2831$ & $\begin{array}{l}\text { Depletes fusion factors from the endosomal } \\
\text { membrane }\end{array}$ & $\underline{79}$ \\
\hline PieE & lpp1953 & $\operatorname{lpg} 1969$ & Binds Rab5 and Rab7 & $\underline{80}$ \\
\hline RidL & lpp2259 & $\operatorname{lpg} 2311$ & Impairs retrograde trafficking & $\underline{83}$ \\
\hline \multicolumn{5}{|c|}{ Bacterial interaction with the ER and LCV formation } \\
\hline \multirow[t]{3}{*}{ SidM (DrrA) } & & \multirow{3}{*}{$\operatorname{lpg} 2464$} & Binds the membrane & 193 \\
\hline & & & Recruits Rab1 to the LCV & $\underline{87}, \underline{88}$ \\
\hline & & & AMPylates Rab1 & $\underline{89}, \underline{90}$ \\
\hline SidD & & $\operatorname{lpg} 2465$ & DeAMPylates Rab1 & $\underline{91}$ \\
\hline LepB & $\operatorname{lpp} 2555$ & $\operatorname{lpg} 2490$ & Converts Rab1 GTP into Rab1 GDP & $\underline{92}$ \\
\hline AnkX & lpp0750 & $\operatorname{lpg} 0695$ & Attaches a phosphocholine moiety to Rab1 & $\underline{93}$ \\
\hline Lem3 & lpp0751 & $\operatorname{lpg} 0696$ & Removes phosphocholination & 94 \\
\hline LidA & lpp1002 & $\operatorname{lpg} 0940$ & Enables the tethering of ER-derived vesicles & $\underline{90}$ \\
\hline
\end{tabular}




\begin{tabular}{|c|c|c|c|c|}
\hline RalF & lpp1932 & $\operatorname{lpg} 1950$ & Recruits Arf1 to the LCV membrane & $\underline{95}$ \\
\hline Ceg9 & lpp0316 & $\operatorname{lpg} 0246$ & Interacts with Rtn4 & 97 \\
\hline LseA & \multicolumn{2}{|c|}{ Corby strain LPC_2110 } & Mediates membrane fusion & 101 \\
\hline LegC3 & lpp1666 & $\operatorname{lpg} 1701$ & Modulates membrane fusion events & $102,102 \mathrm{a}$ \\
\hline LegG1 (MitF) & & $\operatorname{lpg} 1976$ & $\begin{array}{l}\text { Activates Ran GTPase; implicated in } \\
\text { mitochondrial fragmentation }\end{array}$ & 103,104 \\
\hline \multicolumn{5}{|c|}{ Establishing a safe niche: hijacking the host cell response } \\
\hline \multicolumn{5}{|c|}{ Autophagic response } \\
\hline \multirow[t]{2}{*}{ RavZ } & & $\operatorname{lpg} 1683$ & Irreversibly deconjugates LC3 & 107 \\
\hline & lpp1139 & $\operatorname{lpg} 1137$ & Cleaves syntaxin 17 & 108 \\
\hline$L p \mathrm{SPL}$ & lpp2128 & $\operatorname{lpg} 2176$ & Prevents autophagosome formation & $\underline{109}$ \\
\hline \multicolumn{5}{|c|}{ Kinase signaling } \\
\hline LeSHs & \multicolumn{2}{|c|}{11 different effectors } & Bind to phosphorylated Tyr & $\underline{110}$ \\
\hline LegK7 & lpp1899 & $\operatorname{lpg} 1924$ & Targets the Hippo pathway & $\underline{111}$ \\
\hline LegK1 & lpp1439 & $\operatorname{lpg} 1483$ & Activates NF- $\mathrm{kB}$ & $\underline{113}$ \\
\hline LnaB & lpp2592 & $\operatorname{lpg} 2527$ & Activates NF- $\mathrm{kB}$ & 114 \\
\hline MavC & lpp2086 & $\operatorname{lpg} 2147$ & Dampens NF-кB signaling & $\underline{115}$ \\
\hline Lgt1 & lpp1322 & $\operatorname{lpg} 1368$ & Decrease production of I $\mathrm{I} B$, an inhibitor of & $\underline{116}$ \\
\hline Lgt2 & & $\operatorname{lpg} 2862$ & the NF-kB pathway & \\
\hline
\end{tabular}




\begin{tabular}{|c|c|c|c|c|}
\hline Lgt3 & lpp1444 & $\operatorname{lpg} 1488$ & & \\
\hline SidI & lpp2572 & $\operatorname{lpg} 2504$ & & \\
\hline SidL & lpp0504 & $\operatorname{lpg} 0437$ & & \\
\hline Ceg4 & lpp0110 & $\operatorname{lpg} 0096$ & Impacts MAPK signaling & $\underline{120}$ \\
\hline \multicolumn{5}{|c|}{ Epigenetic regulation } \\
\hline RomA & lpp1683 & $\operatorname{lpg} 1718$ & Changes histone marks & $\underline{121}$ \\
\hline \multicolumn{5}{|c|}{ mRNA processing } \\
\hline SnpL & lpp2587 & $\operatorname{lpg} 2519$ & Regulates mRNA processing & $\underline{122}$ \\
\hline \multicolumn{5}{|c|}{ Ubiquitin pathway } \\
\hline LubX & lpp2887 & $\operatorname{lpg} 2830$ & E3 ligase; targets Clk1 & $\underline{123}$ \\
\hline GobX & lpp2521 & $\operatorname{lpg} 2455$ & E3 ligase; locates to Golgi membranes & $\underline{124}$ \\
\hline RavN & lpp1112 & $\operatorname{lpg} 1111$ & E3 ligase & $\underline{125}$ \\
\hline SidC & lpp2579 & $\operatorname{lpg} 2511$ & E3 ligase; phagosomal remodeling & $\underline{127}$ \\
\hline LegU1 & lpp0233 & $\operatorname{lpg} 0171$ & \multirow{2}{*}{ F-box domain } & \multirow[t]{2}{*}{$\underline{128}$} \\
\hline LicA & lpp1363 & $\operatorname{lpg} 1408$ & & \\
\hline AnkB & lpp2082 & $\operatorname{lpg} 2144$ & $\begin{array}{l}\text { F-box domain; ubiquitinates ParvB and } \\
\text { supplies nutrients to the vacuole }\end{array}$ & $\underline{128-130}$ \\
\hline SidE & lpp0304 & $\operatorname{lpg} 0234$ & $\begin{array}{l}\text { Ubiquitinate ER-associated Rab GTPases } \\
\text { and target Rtn4 to control tubular ER }\end{array}$ & $\underline{131}, \underline{132}$, \\
\hline
\end{tabular}




\begin{tabular}{|c|c|c|c|c|}
\hline SdeA & lpp2096 & $\operatorname{lpg} 2157$ & \multirow[t]{3}{*}{ dynamics } & \multirow[t]{3}{*}{$\underline{134}$} \\
\hline SdeB & lpp2095 & $\operatorname{lpg} 2156$ & & \\
\hline $\mathrm{SdeC}$ & lpp2092 & $\operatorname{lpg} 2153$ & & \\
\hline SidJ & lpp2094 & $\operatorname{lpg} 2155$ & Reverses SidE family activity & $\underline{133}$ \\
\hline LotA (Lem 21) & lpp2202 & $\operatorname{lpg} 2248$ & Cleaves ubiquitin from the LCV & $\underline{135}$ \\
\hline \multicolumn{5}{|c|}{ Modulation of cell death } \\
\hline SidF & lpp2637 & $\operatorname{lpg} 2584$ & Antagonizes proapoptotic Bcl-rambo & $\underline{137}$ \\
\hline $\operatorname{SdhA}$ & lpp0443 & $\operatorname{lpg} 0376$ & Prevents cell death & $\underline{138}$ \\
\hline & lpp0782 & $\operatorname{lpg} 0716$ & \multirow{5}{*}{ Induce proapoptotic caspase- 3 activity } & \multirow[t]{5}{*}{$\underline{139}$} \\
\hline Ceg18 & lpp0959 & $\operatorname{lpg} 0898$ & & \\
\hline Lem12 & lpp1595 & $\operatorname{lpg} 1625$ & & \\
\hline $\begin{array}{l}\operatorname{LegS} 2 \\
\end{array}$ & $\operatorname{lpp} 2128$ & $\operatorname{lpg} 2176$ & & \\
\hline VipD & lpp2888 & $\operatorname{lpg} 2831$ & & \\
\hline
\end{tabular}

Abbreviations: ER, endoplasmic reticulum; LCV, Legionella-containing vacuole; MAPK, mitogen-activated protein kinase.

${ }^{a}$ Empty cells in the Effector column indicate that no specific name was given to the effector other than the gene name.

${ }^{b}$ Empty cells in the Gene column indicate that there is no orthologous gene in the Legionella species. 


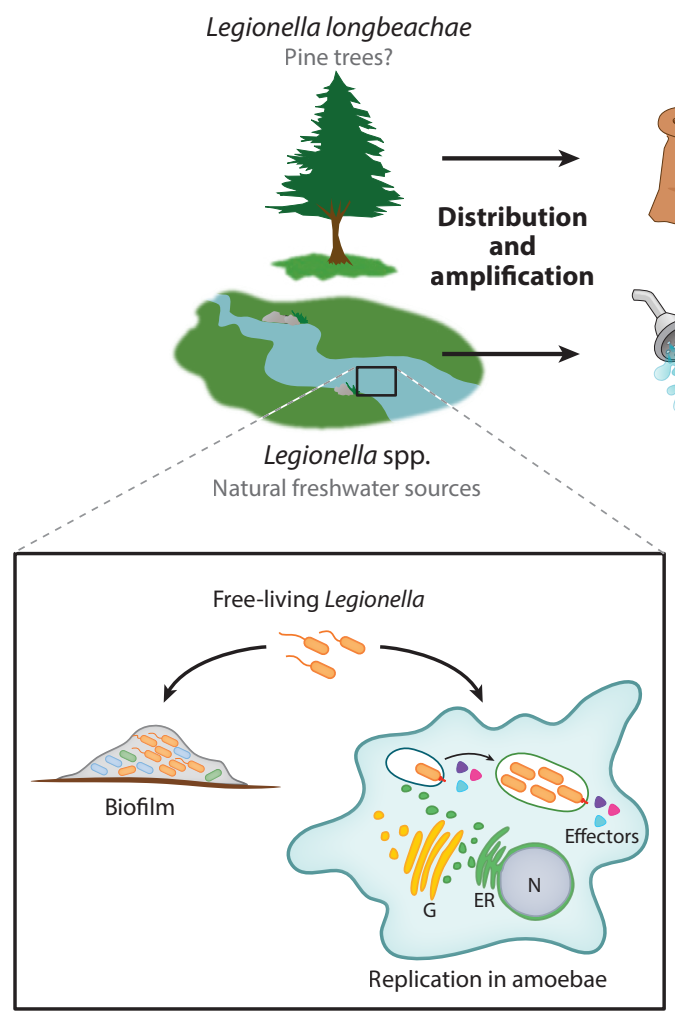

Colonization

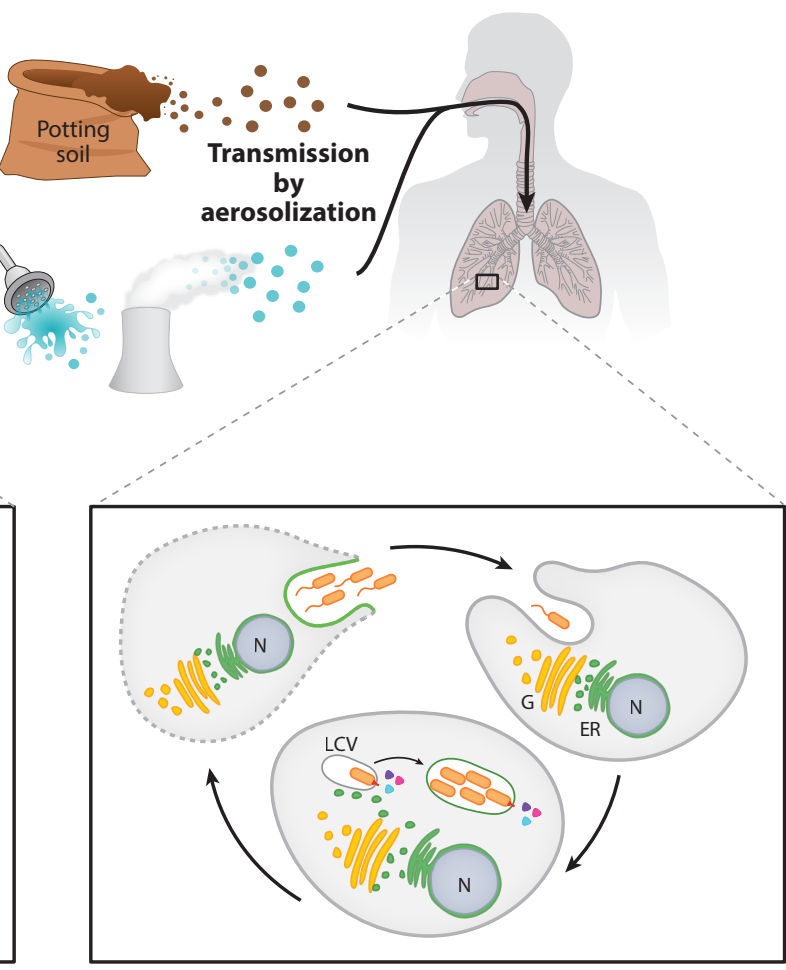

Macrophage infection 


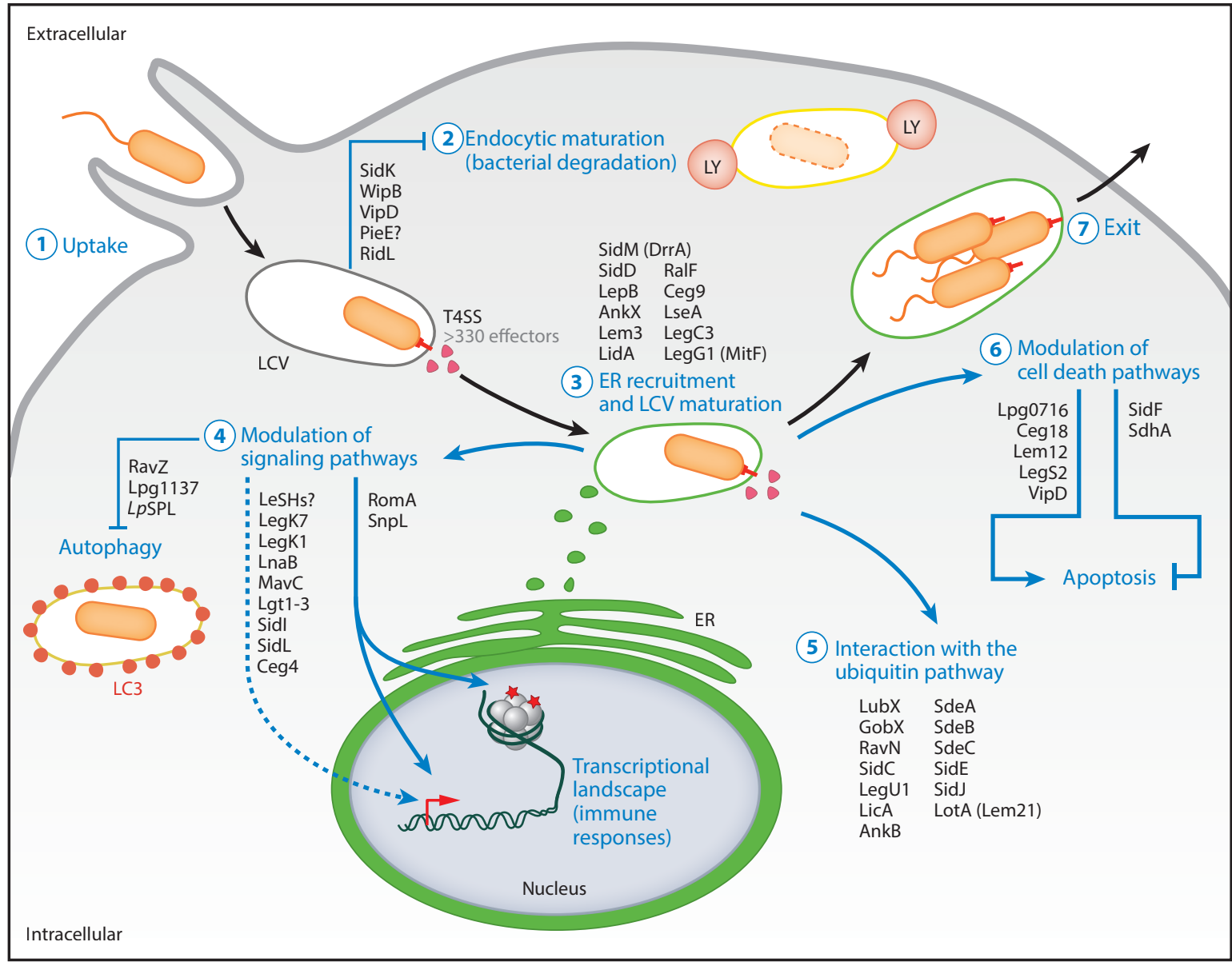



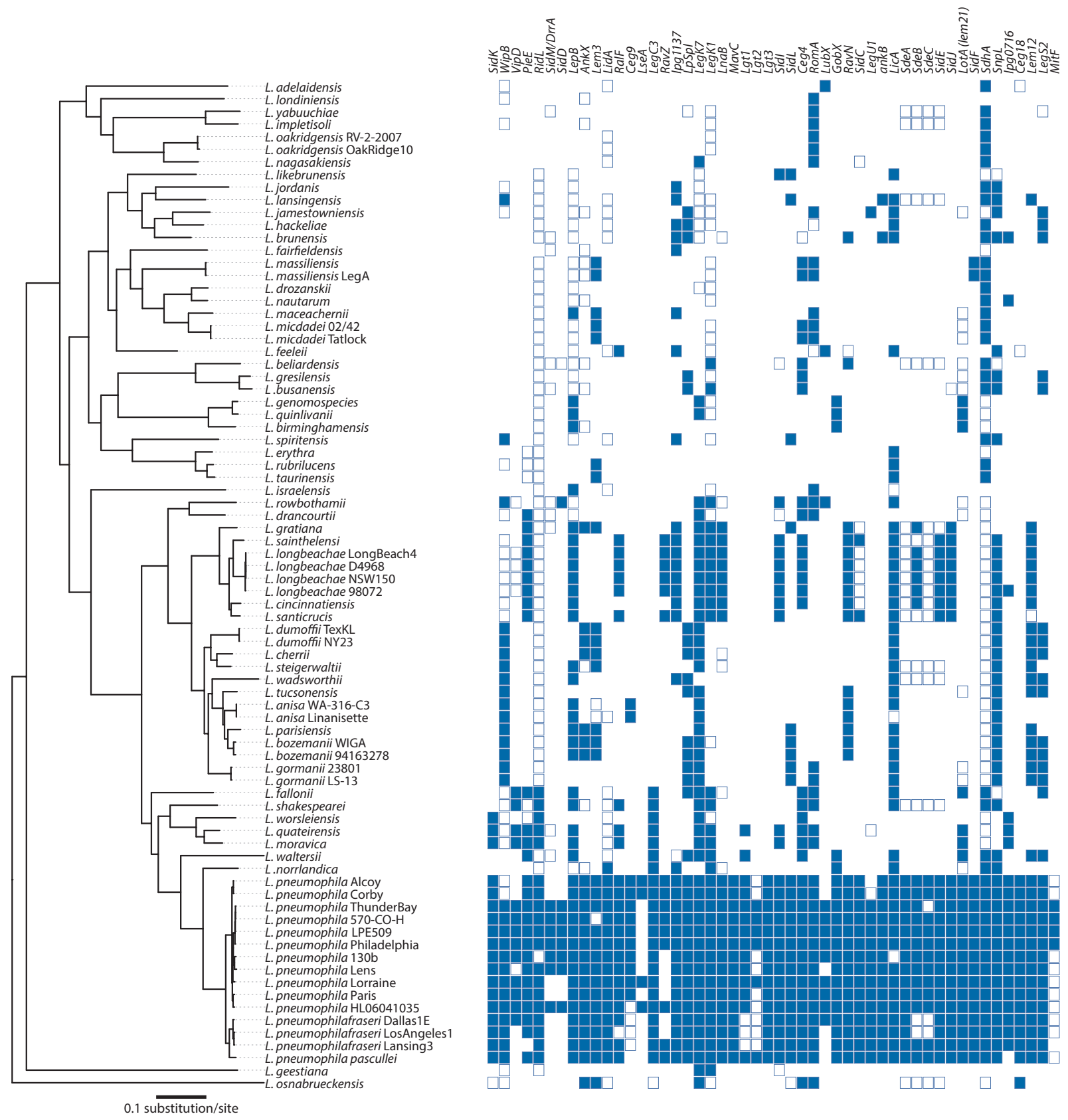


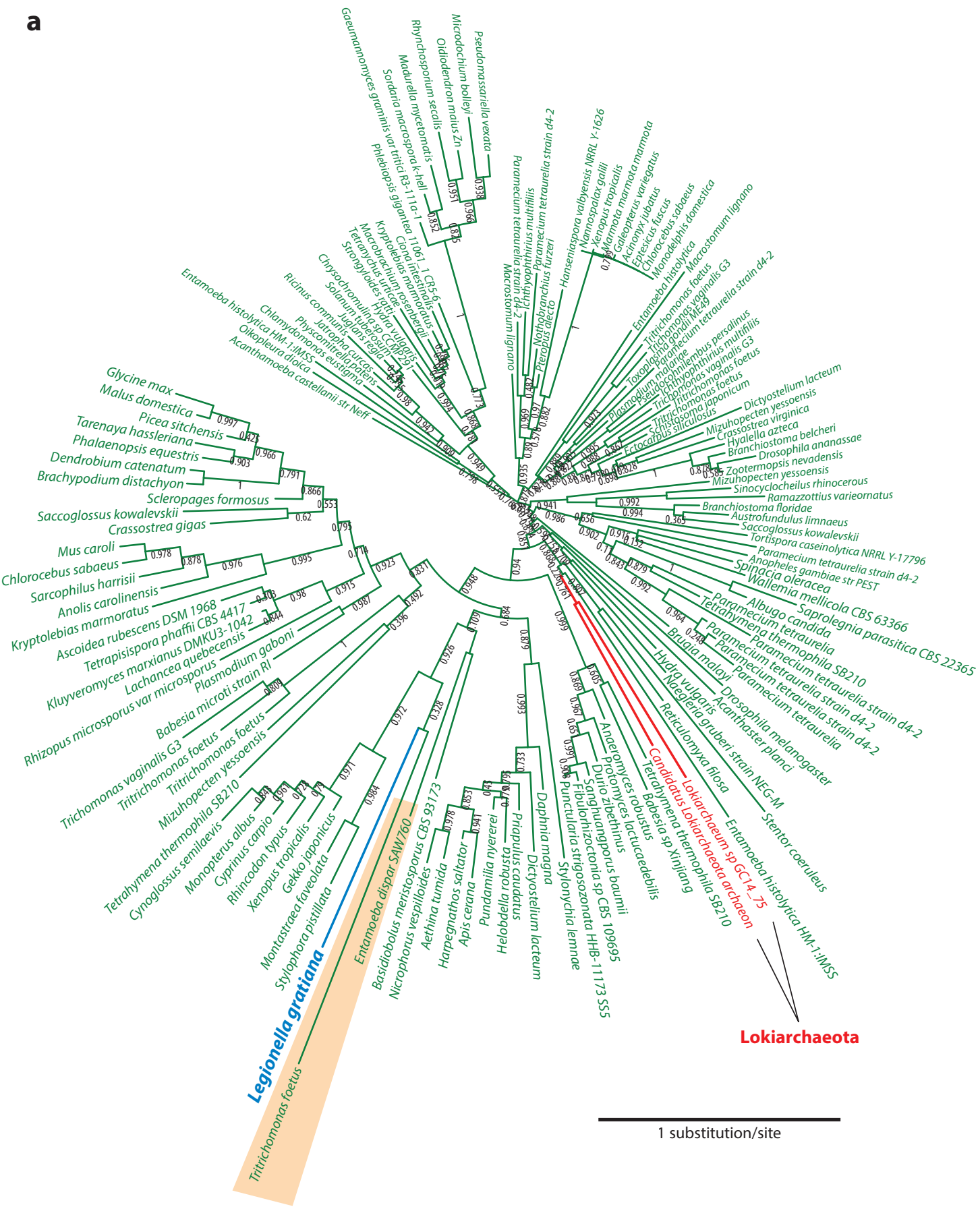

Protists 
b

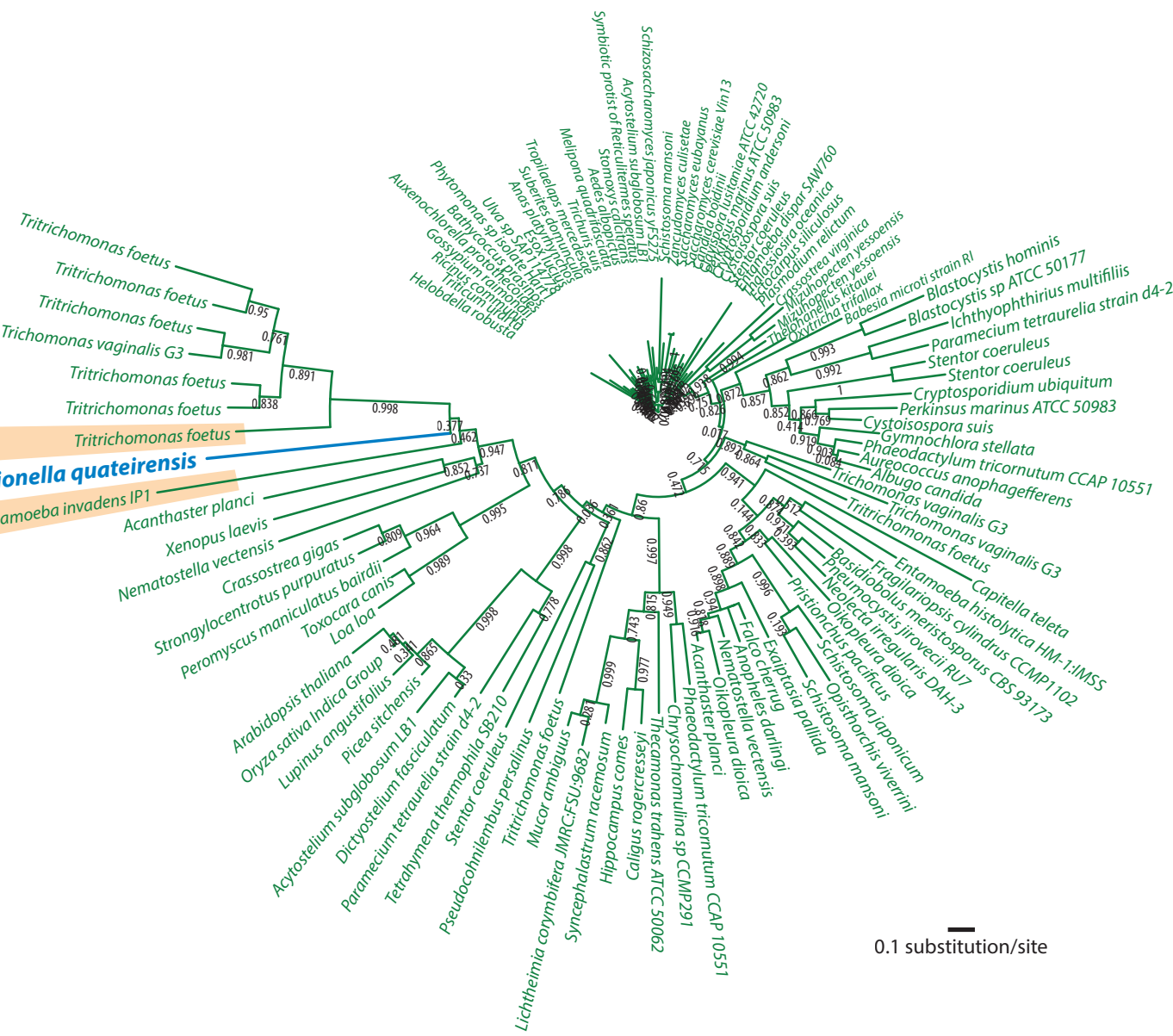

